\title{
Induction of vaginal-resident HIV-specific CD8 T cells with mucosal prime-boost immunization
}

\author{
H-X Tan ${ }^{1}$, AK Wheatley ${ }^{1,2}$, R Esterbauer $^{1}$, S Jegaskanda ${ }^{1}$, JJ Glass ${ }^{1,2}$, D Masopust ${ }^{3,4}$, R De Rose ${ }^{1,5,7}$ and \\ SJ Kent ${ }^{1,2,6,7}$
}

Tissue-resident memory $\left(T_{\mathrm{RM}}\right)$ CD8 T cells survey a range of non-lymphoid mucosal tissues where they rapidly mediate clearance of viral infections at the entry portals. Vaccines that establish CD8 $\mathrm{T}_{\mathrm{RM}}$ cells in the cervicovaginal mucosa hold promise for effective immunity against sexually transmitted HIV. We demonstrate that HIV-specific CD8 $T_{R M}$ cells can be established in the murine vaginal mucosa using a combined intranasal and intravaginal mucosal immunization with recombinant influenza-HIV vectors. Using in situ tetramer immunofluorescence microscopy, we found that this mucosally administered prime-boost immunization also resulted in the durable seeding of CD8 T cells in the frontline vaginal epithelial compartment as opposed to the vaginal submucosa. Upon cognate antigen recognition within the vaginal mucosa, these HIV-specific CD8 $\mathrm{T}_{\mathrm{RM}}$ cells rapidly initiated a tissue-wide state of immunity. The activation of HIVspecific CD8 $T_{\mathrm{RM}}$ cells resulted in the upregulation of endothelial vessel addressin expression and substantial recruitment of both adaptive and innate immune cells in the vaginal mucosa. These findings suggest that the epithelial localization of HIV-specific CD8 $\mathrm{T}_{\mathrm{RM}}$ cell populations and their capacity to rapidly activate both arms of the immune system could significantly augment frontline defenses against vaginal HIV infection.

\section{INTRODUCTION}

HIV infection in women is primarily acquired across the cervicovaginal mucosa and there is a critical need for vaccines that induce immunity at this portal of entry. Early HIV infection can occur in intraepithelial CD4 T cells, and parallel Simian immunodeficiency virus models provide evidence that infection is spatially restricted to submucosal CD4 T cells for a few critical days before systemic dissemination ensues. ${ }^{1-3}$ This brief period of localized infection in the cervicovaginal tissue represents a potential window of opportunity for rapid immune intervention by tissue-resident memory $\left(\mathrm{T}_{\mathrm{RM}}\right) \mathrm{CD} 8 \mathrm{~T}$ cells if they can be generated in the genital tissues by vaccination.

T-cell subsets have conventionally been described as either central memory $\left(\mathrm{T}_{\mathrm{CM}}\right)$ or effector memory $\left(\mathrm{T}_{\mathrm{EM}}\right)$ based on their ability to continually migrate into secondary lymphoid organs or non-lymphoid tissues, respectively. ${ }^{4}$ In contrast, $T_{R M}$ populations remain in non-lymphoid tissues long after the resolution of infection and exist in disequilibrium with migratory T-cell populations. ${ }^{5-11}$ Unlike $\mathrm{T}_{\mathrm{EM}}$ and $\mathrm{T}_{\mathrm{CM}}$ cells, $\mathrm{T}_{\mathrm{RM}}$ cells are also commonly positioned at the frontline barrier of non-lymphoid tissues. ${ }^{6,12} \mathrm{~T}_{\mathrm{RM}}$ cells locally survey tissues for infected cells and initiate tissue-wide inflammation upon detection of cognate antigen, thus enhancing viral clearance. ${ }^{13-16}$ Recent studies have unequivocally demonstrated the critical functions of $\mathrm{T}_{\mathrm{RM}}$ cells in protecting mucosal tissues against infection by a range of viral pathogens including HSV,${ }^{17,18}$ influenza, ${ }^{19}$ and respiratory syncytial virus. ${ }^{20}$

Large-scale T-cell vaccine efforts, including the STEP, Phambili, and HVTN505 trials in the recent decade have failed to demonstrate protective efficacy against sexually transmitted HIV. ${ }^{21-24}$ Despite observable systemic T-cell responses induced by these vaccine regimens and some evidence of a "sieving" effect on viral strains acquired by the vaccinees, ${ }^{25,26}$ it is likely that immediate clearance of

${ }^{1}$ Department of Microbiology and Immunology, The University of Melbourne at the Peter Doherty Institute for Infection and Immunity, Melbourne, Victoria, Australia. ${ }^{2}$ ARC Centre of Excellence in Convergent Bio-Nano Science and Technology, The University of Melbourne, Melbourne, Victoria, Australia. ${ }^{3}$ Department of Microbiology and Immunology, University of Minnesota, Minneapolis, Minnesota, USA. ${ }^{4}$ Center for Immunology, University of Minnesota, Minneapolis, Minnesota, USA. ${ }^{5}$ ARC Centre of Excellence in Convergent Bio-Nano Science and Technology, Monash University, Parkville, Victoria, Australia and ${ }^{6}$ Melbourne Sexual Health Centre, Alfred Hospital, Monash University Central Clinical School, Melbourne, Victoria, Australia. Correspondence: SJ Kent (skent@unimelb.edu.au)

${ }^{7}$ These authors are joint last authors. 
infection in mucosal tissues would be inefficient given the temporal delay in anamnestic recall of systemic memory T-cell responses and dissemination of effector cells to the site of infection. ${ }^{27,28}$ Effective T-cell immunity against viruses at their entry points will likely require local seeding of mucosal tissue with CD8 T cells. In macaques vaccinated by attenuated CMV vectors, persistent induction and wide distribution of CD8 $\mathrm{T}_{\mathrm{EM}}$ cells in effector sites, including mucosal tissues, provided immunity against Simian immunodeficiency virus infection in $\sim 50 \%$ of vaccinated animals. ${ }^{29,30}$ In a subset of women highly exposed to HIV-1 that remained uninfected, HIV-specific CD8 $\mathrm{T}$ cells can be detected in the female reproductive tissues. ${ }^{31}$ These studies allude to the importance of establishing CD8 $\mathrm{T}$-cell immunity at the frontline niche of mucosal tissues to mount an effective and rapid response against genital HIV infection.

Here we demonstrate that HIV-specific CD8 $\mathrm{T}_{\mathrm{RM}}$ cells can be established in the vaginal mucosa of mice using mucosal vaccination strategies. Vaccination using recombinant influenza-HIV vectors resulted in the substantial shifting of HIVspecific CD8 T cells from the submucosa into the epithelium of the vaginal mucosa, thus priming the tissue against subsequent infection at the frontline barrier. Once established, we show that these cells can recognize vaginally administered HIV antigen and rapidly modulate inflammation in the vaginal mucosa. Antigen detection resulted in the upregulation of tissue addressin expression and the activation of a vaginal recruitment cascade for both adaptive and innate immune cells. The seeding of CD8 $\mathrm{T}_{\mathrm{RM}}$ cells into the vaginal mucosa, specifically in the epithelium compartment, may provide a rapid first line of defense against vaginal HIV infection.

\section{RESULTS \\ Intravaginal boosting induces vaginal mucosal HIV-specific CD8 T cells}

We recently developed recombinant influenza viruses expressing the HIV-1 Gag protein p24. ${ }^{32}$ To induce CD8 T-cell immunity in the vaginal mucosa, a immunization regimen consisting of an intranasal prime followed by an intravaginal boost was performed. Intranasal immunization can prime cytotoxic $\mathrm{T}$ lymphocyte responses in the vaginal mucosa, which can be subsequently boosted by vaginal immunization. ${ }^{33}$ The latter route of immunization results in more potent and sustained immunity in the vaginal mucosa. ${ }^{34-36}$ Mice were sequentially immunized with an intranasal Flu(PR8)-p24 prime and an intravaginal $\mathrm{Flu}(\mathrm{X} 31)$-p24 boost at doses of $10^{5}$ and $10^{7}$ p.f.u., respectively (Figure 1a).

Before analyzing whole tissues by immunofluorescence microscopy, we conducted flow cytometric analyses to confirm that this immunization induced HIV-1 p24-specific CD8 T cells at this effector phase of 14 days after boost. Blood samples were analyzed for HIV-specific CD8 T-cell responses using a H2-K ${ }^{\mathrm{D}}$ tetramer loaded with the AI9 epitope of the p24 protein (Figure 1b, top row). Mock-immunized mice lacked HIVspecific CD8 T cells. In mice immunized with the Flu-p24 vectors, HIV-specific CD8 T-cell responses were readily detected in blood. We next analyzed mucosal samples to assess how this regimen effectively primed responses in the lung and vagina. HIV-specific CD8 T cells were readily detectable in lung bronchoalveolar lavage (BAL) samples 14 days after boost (Figure 1b, middle row). Cell suspensions of vaginal tissues were analyzed at 14 days after boost, as per the gating strategy shown in Supplementary Figure S1A online. HIV-specific CD8 T-cell responses in the vaginal mucosa were comparable to responses observed in lung BAL samples (Figure $\mathbf{1 b}$, bottom row). Mice immunized with influenza vector controls lacking HIV-1 p24 expression did not induce vaginal HIV-specific CD8 T-cell responses (Supplementary Figure S1B,C). Overall, HIV-specific CD8 T-cell responses were significantly elevated in Flu-p24-immunized mice for all tissues investigated vs. mock-immunized mice (Figure 1d), and are consistent with previous observations. ${ }^{32}$

CD103, a marker of tissue residency, was examined in all tissue compartments (Figure 1c,e). CD103 expression was significantly increased in HIV-specific CD8 T cells of the vaginal mucosa compared with lung BAL samples, and was negligible in blood. These data demonstrated that an intranasal prime followed by an intravaginal boost immunization with the Flu-p24 vectors induced a high level of HIV-specific CD8 $T_{R M}$ cells in the vaginal mucosa and suggested that this immunization model would be suitable for detailed analyses by immunofluorescence microscopy.

\section{In situ tetramer immunofluorescence microscopy as a method to increase detection of HIV-specific CD8 T cells in the vaginal mucosa}

Standard flow cytometry-based assays for non-lymphoid tissue analyses underestimate $\mathrm{T}_{\mathrm{RM}}$ cells by $\sim 70$-fold compared with image-based quantitation. ${ }^{37}$ Moreover, the anatomical localization of immune cells within non-lymphoid tissues is absent in flow cytometry-based assays of tissue suspensions. This information is relevant for $\mathrm{T}_{\mathrm{RM}}$ cells, which preferentially localize to the vaginal epithelium..$^{7,12,17,35,36}$ To address these limitations, we utilized in situ tetramer (IST) immunofluorescence staining to visually detect HIV-specific CD8 T cells in the vaginal mucosa and complement data generated by flow cytometry. ${ }^{38}$ Whole fresh vaginal tissues were stained with the AI9 tetramer before cryopreservation and tissue sectioning, which differs from other published methods utilizing vibratome-based processing ${ }^{39}$ or Qdot staining for equivalent detection of fluorescence. ${ }^{40}$

Vaginal mucosal tissues from mice immunized with the Flup24 viruses were stained with $\mathrm{H} 2-\mathrm{K}^{\mathrm{D}}$ tetramers loaded with an HIV-1 p24 capsid AI9 peptide (AMQMLKETI) or an irrelevant TUM peptide (KYQAVTTTL). Tissues stained with the $\mathrm{K}^{\mathrm{D}}$ AI9 tetramer detected HIV-specific CD8 T cells only in immunized mice (Figure 2a,c). The tetramer specificity for CD8 T cells was confirmed in tissues stained with the $\mathrm{K}^{\mathrm{D}}$ TUM tetramer, with no TUM-specific CD8 $\mathrm{T}$ cells detected (Figure 2b). We observed some nonspecific tetramer staining (red cells, Figure 2c), which is attributed to the interaction of excess phycoerythrin-conjugated streptavidin with fibronectin 
a

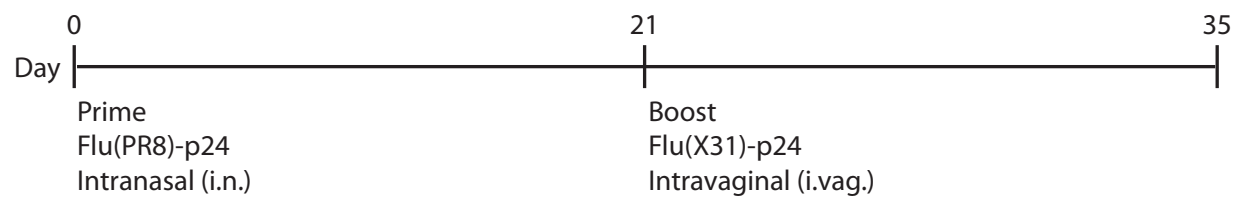

b
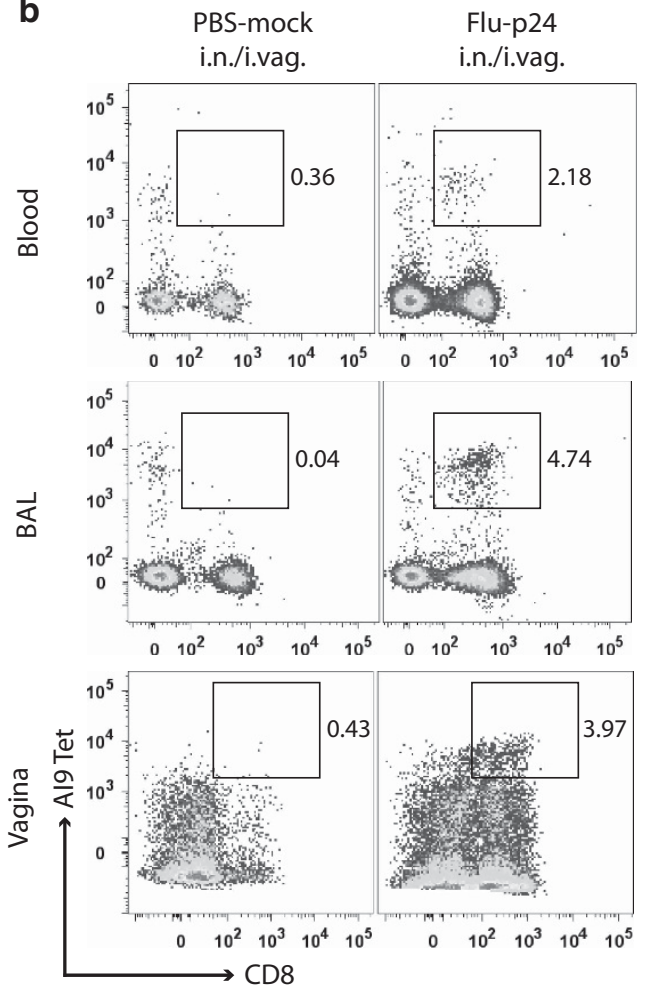

C
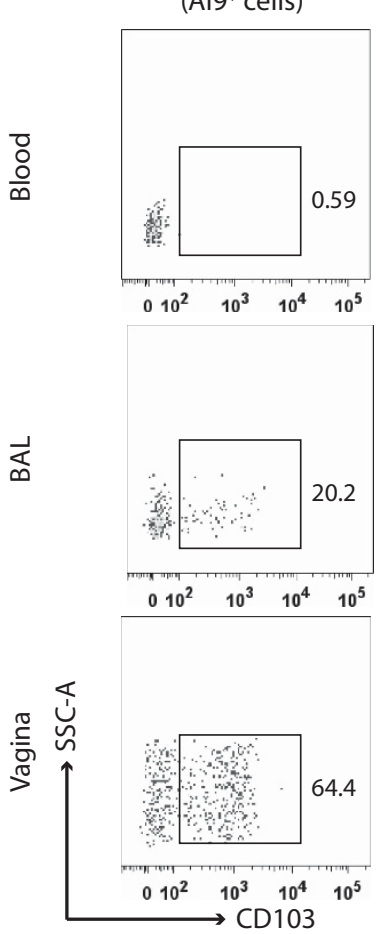

d

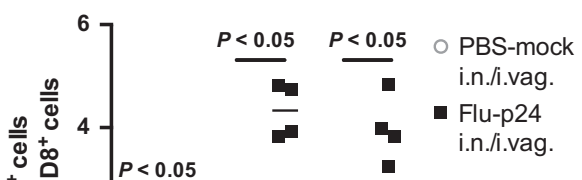

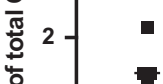

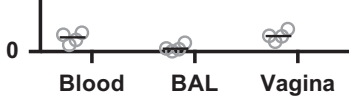

e

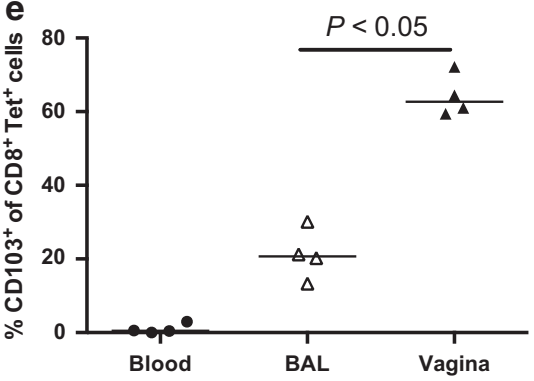

Figure 1 Induction of systemic and mucosal HIV-specific CD8 T-cells by intranasal (i.n.) prime and intravaginal (i.vag.) boost immunizations. (a) Primeboost vaccination with Flu-p24 vectors for the induction of vaginal mucosa HIV-specific CD8 T cells. (b) HIV-specific CD8 T cells in blood (top row), bronchoalveolar lavage (BAL) (middle row) and vaginal mucosa (bottom row) 14 days after boost, and (c) CD103 expression of corresponding HIVspecific CD8 T cells isolated from blood (top), BAL (middle), and vaginal mucosa (bottom) of Flu-p24-immunized mice. Plots are representative of four independent experiments with cells pooled from three mice per treatment group and frequencies are calculated as a proportion of total CD8 ${ }^{+}$cells gated. (d) Frequency of HIV-specific CD8 T cells in blood, BAL, and vaginal mucosa; data points represent responses pooled from three mice per treatment group. (e) Frequency of CD103 expression on HIV-specific CD8 T cells in blood, BAL, and vaginal mucosa; data points represent responses pooled from three mice per treatment group. Bars represent median values, and $P$ values were derived from Mann-Whitney statistical testing.

receptors of non-CD8 T cells. ${ }^{41}$ Background tetramer staining was equivalent in non-CD8 cells stained with either the $\mathrm{K}^{\mathrm{D}}$ AI9 or $\mathrm{K}^{\mathrm{D}}$ TUM control tetramer (Supplementary Figure S2A), confirming binding of excess phycoerythrin-conjugated streptavidin. Therefore, colocalized IST staining of tetramer and CD8 signals allowed accurate quantification and spatial enumeration of HIV-specific CD8 $\mathrm{T}$ cells in the vaginal mucosa.

\section{Intravaginal immunization establishes effector HIV-specific CD8 $\mathrm{T}$ cells in the vaginal epithelium}

We subsequently utilized the IST staining to quantify the immune response in the vaginal mucosa. The response was analyzed as a proportion of all nucleated cells, and the localization of HIV-specific CD8 T cells in either the lamina propria or epithelial compartment was also delineated. Responses in immunized mice were analyzed at the peak of the effector phase 14 days after the last vaccination (Figure 1a).
At the effector phase, immunized mice displayed a significant increase in HIV-specific CD8 T cells in the vaginal mucosa at an average of $0.5 \mathrm{HIV}$-specific CD8 T cells per 1,000 nucleated cells $(P=0.0001$, Figure $3 \mathbf{a})$, confirming the IST staining results in Figure 2b. Conversely, HIV-specific CD8 T cells were not detected in mock-immunized mice. The HIVspecific CD8 $\mathrm{T}$ cells induced by immunization displayed significant spatial skewing toward the epithelial compartment of the vaginal mucosa as opposed to the lamina propria $(P=0.001$, Figure $\mathbf{3 b})$.

The total CD8 T-cell population was also analyzed, as this accounts for potential cytotoxic $\mathrm{T}$ lymphocytes specific for other HIV- or vector-associated epitopes not revealed by the tetramer analysis. Mock-immunized mice displayed a basal level of 1.7 CD8 T cells for every 1,000 nucleated cells of the vaginal mucosa. In contrast, immunized mice displayed a significantly elevated CD8 T-cell population, approaching 20 CD8 T cells per 1,000 nucleated cells $(P<0.0001$, Figure $3 c)$. 
a

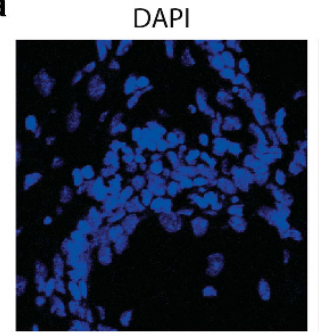

CD8

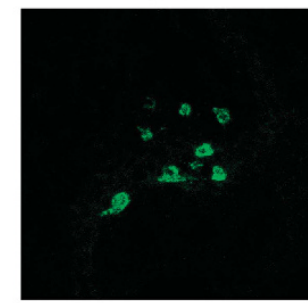

C
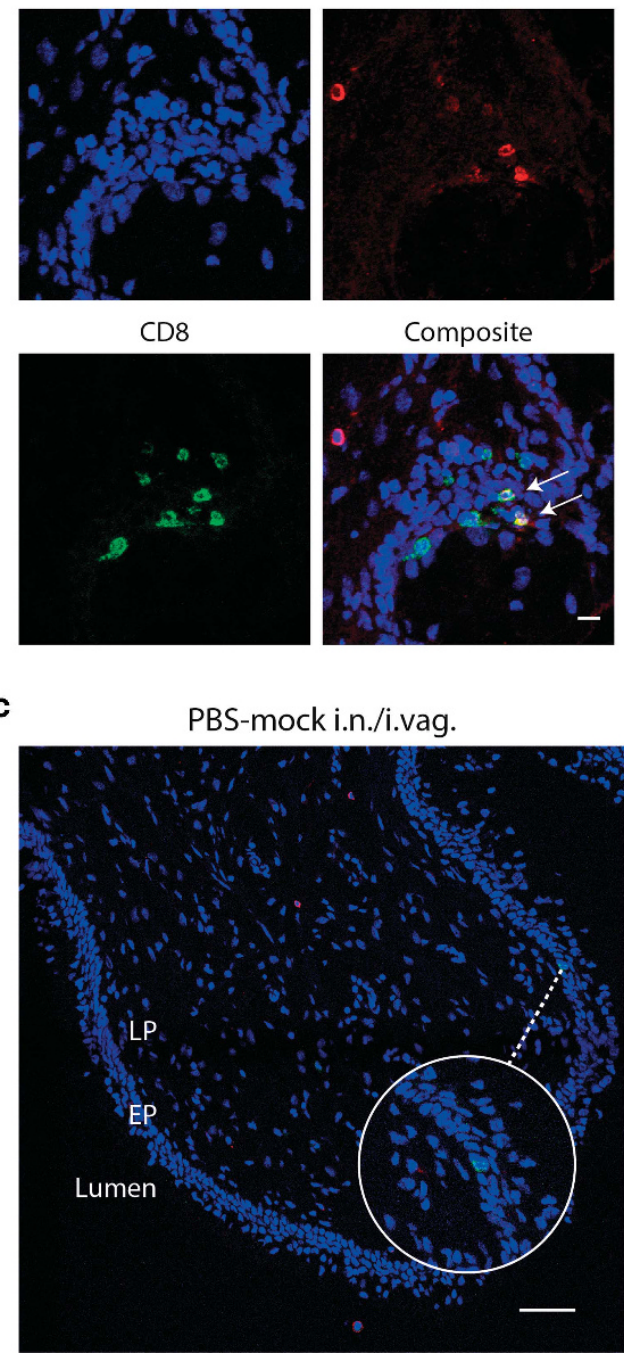

Composite

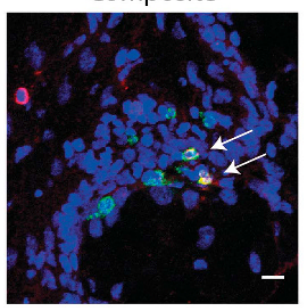

b

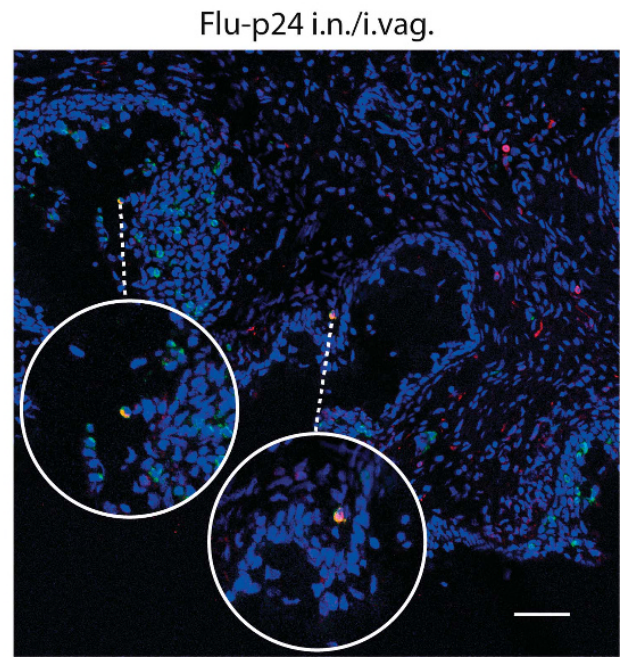

Figure 2 Detection of HIV-specific CD8 T cells in the vaginal mucosa by in situ tetramer immunofluorescence microscopy 14 days after last vaccination with Flu-p24 vectors (intranasal/intravaginal (i.n./i.vag.)). (a) Individual and composite signals for DAPI (4',6-diamidino-2-phenylindole), H2-K ${ }^{\mathrm{D}}$ Al9 tetramer and CD8 T cells, with arrows indicating HIV-specific CD8 T cells. Bar $=10 \mu \mathrm{m}$. (b) Detection of vaginal HIV-specific CD8 T cells in individual mice by use of the AI9 tetramer or an irrelevant TUM tetramer; bars represent median value and graph represents data combined from two experiments $(n=9$ mice per treatment group). (c) Representative vaginal tissue from a mock- or Flu-p24-immunized mouse showing total CD8 T cells (green cells) and epithelium localization of HIV-specific CD8 T cells (red/green composite cells). Bar $=50 \mu \mathrm{m}$. Ep, epithelium; LP, lamina propria.

The spatial localization of CD8 $\mathrm{T}$ cells was analyzed (Figure 3d). Total CD8 T-cell localization of immunized mice displayed a significant bias $(P<0.0001)$ towards the epithelium compartment, similar to that observed for the HIVspecific CD8 T cells. Conversely, mock-immunized mice showed more CD8 $\mathrm{T}$ cells in the lamina propria of the vaginal mucosa than in the epithelium $(P<0.0001)$. The mucosal prime-boost immunization regimen, therefore, induced infiltration of both HIV-specific and total CD8 T-cells into the vaginal mucosa, which displayed a propensity to localize in the epithelium compartment.

\section{Intravaginal immunization establishes memory HIV-specific CD8 T cells in the vaginal mucosa}

Having established that HIV-specific CD8 T cells can be seeded into the vaginal epithelium, we next assessed the durability of this response. In studies investigating $\mathrm{T}_{\mathrm{RM}}$ lodgement in the skin or vaginal mucosa, $C D 8 \mathrm{~T}$ cells displayed $\mathrm{T}_{\mathrm{RM}}$ phenotypes and fixed epithelial compartmentalization by week 4 after infection and beyond. 6,12,17,36 To investigate the HIV-specific CD8 T-cell memory response, immunized mice were analyzed 30 days after the last vaccination (Figure $\mathbf{4 a}$ ).

At this memory time point, most immunized mice retained vaginal HIV-specific CD8 T cells at an average of 0.26 cells for every 1,000 nucleated cells $(P<0.0001$, Figure $4 \mathbf{b})$. In contrast, HIV-specific CD8 T cells were not detected in mockimmunized mice. The distribution of memory HIV-specific CD8 $\mathrm{T}$ cells between the epithelium and lamina propria was similar (Figure 4c).

Total CD8 $\mathrm{T}$ cells remained significantly elevated in the vaginal mucosa 30 days after the last vaccination $(P<0.0001$, Figure 4d), maintaining a sixfold increase compared with 
a
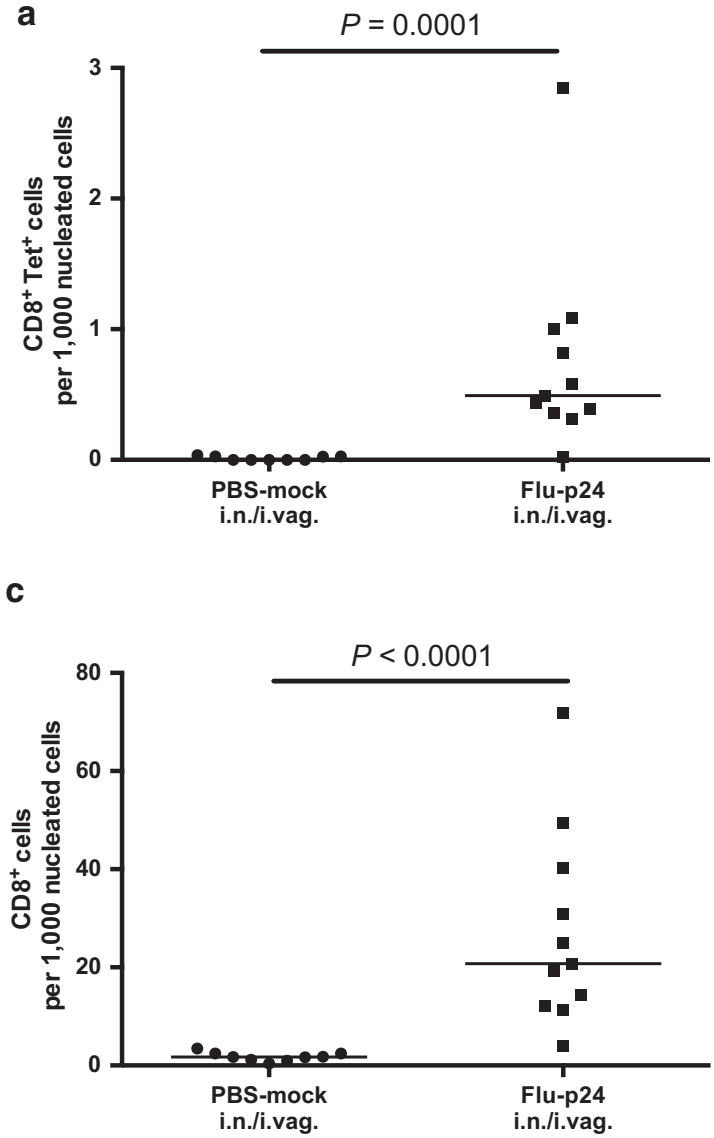

b

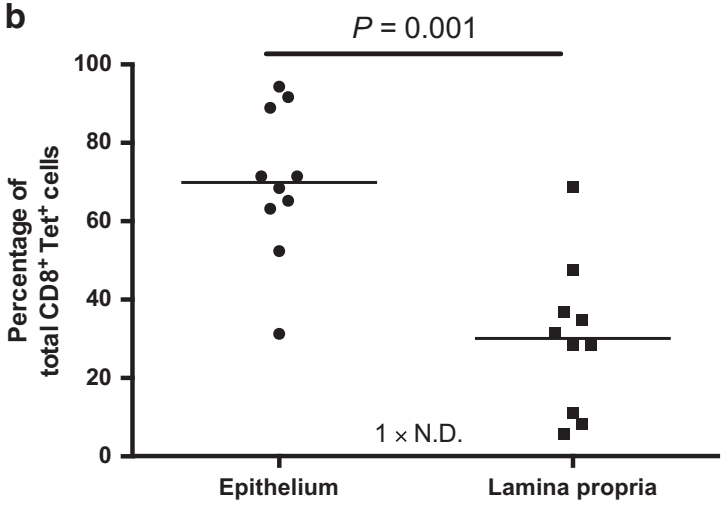

d

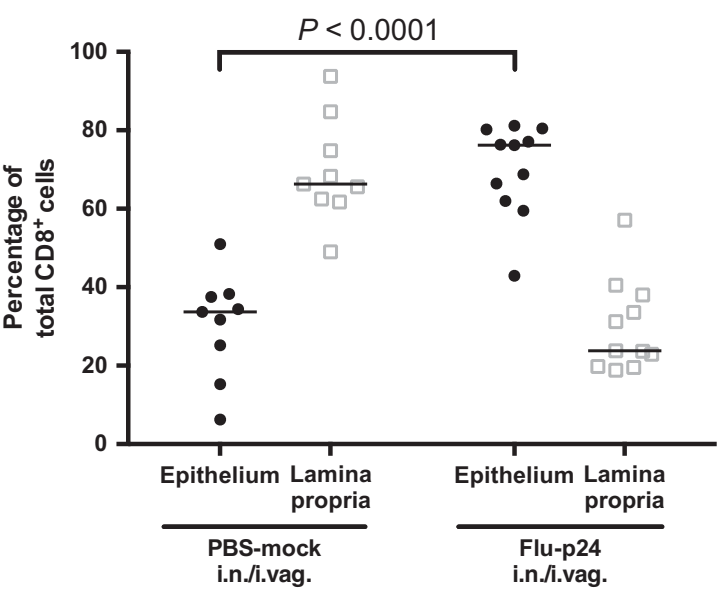

Figure 3 Intravaginal (i.vag.) immunization establishes effector HIV-specific CD8 T cells in the vaginal mucosa 14 days after the last vaccination. (a) Quantification of HIV-specific CD8 T cells in the vaginal mucosa. (b) Spatial distribution of HIV-specific CD8 T cells in the vaginal mucosa; number $\times$ N.D. - mice with insufficient HIV-specific CD8 T cells that could be evaluated. (c) Quantification of total CD8 T cells in the vaginal mucosa. (d) Spatial distribution of total CD8 T cells in the vaginal mucosa. Graphs represent data combined from two experiments $(n=9-11$ mice per treatment group), bars represent median values, and $P$ values were derived from Mann-Whitney statistical testing. i.n., intranasal; ND, not detected; PBS, phosphate-buffered saline.

mock-immunized mice. Mirroring the response at 14 days after the last vaccination, total vaginal CD8 T cells of immunized mice displayed a significant bias $(P<0.05)$ in spatial localization toward the epithelium compartment 30 days after the last immunization (Figure 4e). In contrast, mockimmunized mice maintained a significant preferential skewing $(P<0.05)$ of CD8 T cells toward the lamina propria of the vaginal mucosa. Lamina propria CD8 $\mathrm{T}$ cells in mockimmunized mice at the memory phase were reduced compared with mock-immunized mice at the effector phase (Figure 3d vs. Figure 4e) because of potential waning of progesterone administered before immunization, which decreases vaginal CD8 T-cell infiltration as described previously. ${ }^{42}$ Thus, both HIV-specific and total CD8 T cells were retained in the vaginal mucosa 4 weeks after boost, of which a proportion were spatially maintained in the epithelium.

\section{Maintenance of HIV-specific CD8 T cells between the effector and memory phase}

We investigated the level of waning in vaginal CD8 T cells and if there was a preferential retention of HIV-specific CD8 T cells over the total CD8 $\mathrm{T}$ cells in the vaginal mucosa. As immunizations for the peak effector (14 days after boost) and memory (30 days after boost) phase were initiated concurrently, HIV-specific and total CD8 T-cell responses of both phases could be directly compared. Total CD8 T cells decreased significantly by 4.2 -fold as the effector phase progressed to the memory phase $(P<0.001$, Figure 5a). Concurrently, absolute counts of HIV-specific CD8 T cells were reduced by 1.9-fold from the effector to memory phase progression, although this was not significant $(P=0.24$, Figure 5b). HIV-specific CD8 $\mathrm{T}$ cells as a percentage of total CD8 T cells increased by 2.5-fold from effector to memory phase, but was not significant $(P=0.30$, Figure $5 c)$. Despite a significant decrease in total CD8 $\mathrm{T}$ cells within the vaginal mucosa, $\mathrm{HIV}$-specific CD8 $\mathrm{T}$ cells did not display a similar extent of reduction in both absolute and percentage terms.

The tissue-residency marker, CD103, promotes CD8 T-cell retention in the vaginal mucosa and has been associated with improved control of viral infections. ${ }^{17,43}$ We compared CD103 expression levels in HIV-specific CD8 T-cells by flow 

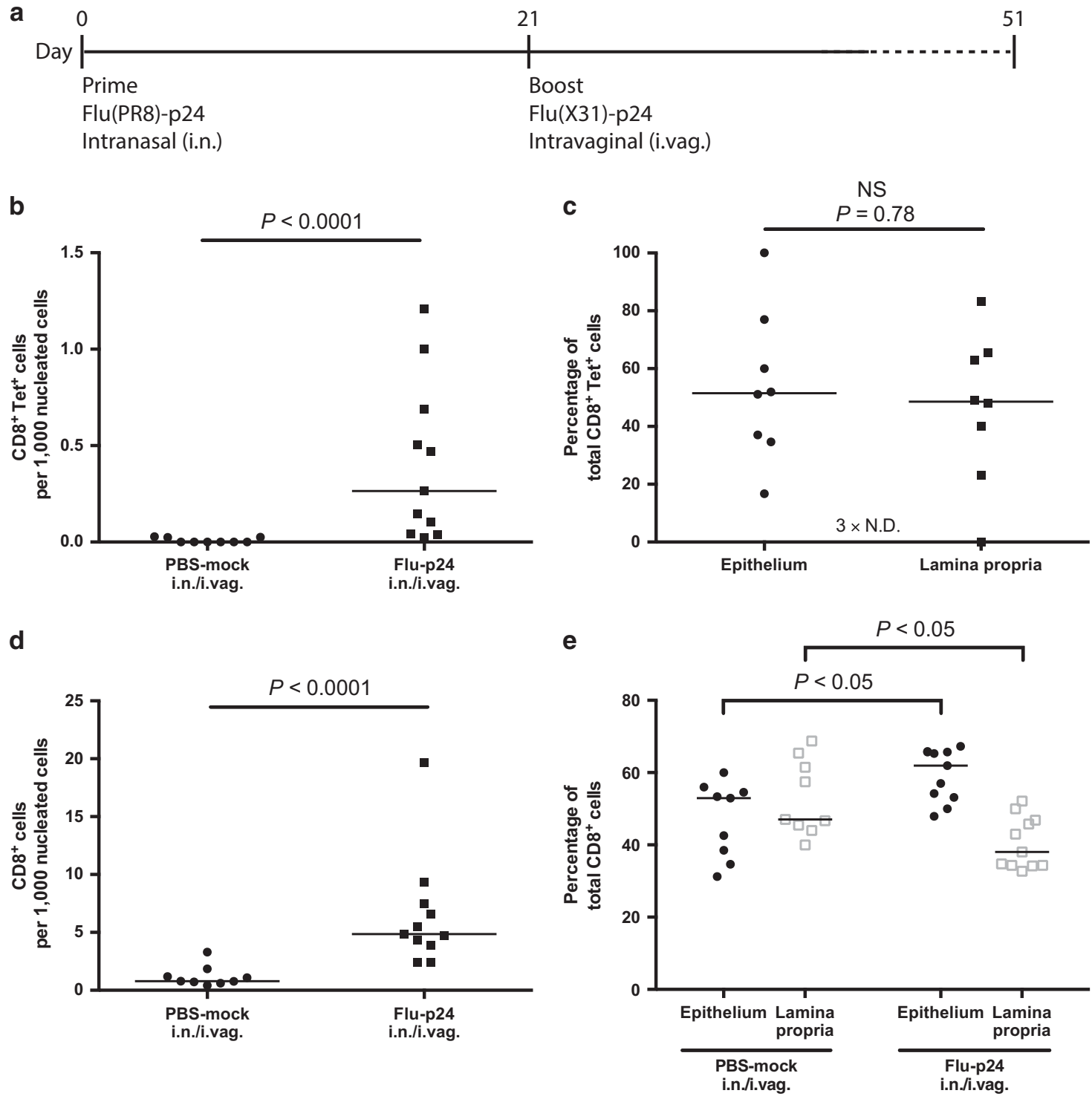

Figure 4 Intravaginal immunization establishes memory HIV-specific CD8 T cells in the vaginal mucosa 30 days after the last vaccination. (a) Primeboost vaccination regimen for the induction of memory HIV-specific CD8 T cells. (b) Quantification of HIV-specific CD8 T cells in the vaginal mucosa. (c) Spatial distribution of HIV-specific CD8 T cells in the vaginal mucosa; number $\times$ N.D.-mice with insufficient HIV-specific CD8 T cells that could be evaluated. (d) Quantification of total CD8 T cells in the vaginal mucosa. (e) Spatial distribution of total CD8 T cells in the vaginal mucosa. Graphs represent data combined from two experiments ( $n=9-11$ mice per treatment group), bars represent median values, and $P$ values were derived from Mann-Whitney statistical testing. ND, not detected; NS, not significant; PBS, phosphate-buffered saline.

cytometry at the peak effector and memory phase (Figure 5d,e). High levels of $\mathrm{CD} 103$ expression were maintained with no significant reduction from the effector to memory phase. To complement the flow cytometry data, we investigated the colocalized expression of CD103 on HIVspecific CD8 $\mathrm{T}$ cells at the memory phase by confocal microscopy (Figure 5f). HIV-specific CD8 $\mathrm{T}$ cells present in the epithelial compartment displayed CD103 expression, and conversely, CD103 expression was absent for HIV-specific CD8 $\mathrm{T}$ cells localized in the lamina propria (Figure 5g). These observations suggest that HIV-specific CD8 T cells induced by immunization displayed tissue-resident properties early during the effector phase and were maintained later in the memory phase.
In vivo reactivation of vaginal-resident HIV-specific CD8 $T$ cells induces infiltration of peripheral CD8 T cells

$\mathrm{T}_{\mathrm{RM}}$ cells function as frontline sensors in non-lymphoid tissues such as the vaginal mucosa and can initiate rapid recruitment of peripheral immune cells upon antigen-specific activation. ${ }^{8,14}$ As a model to assess the functional capacity of vaginal-resident memory HIV-specific CD8 T cells, we inoculated immunized animals intravaginally with either the HIV AI9 peptide (AMQMLKETI) or an irrelevant LYL peptide (LYLVCGERL), and tissue-wide activation was assessed 2 days later (Figure 6a).

Reactivation of HIV-specific CD8 $\mathrm{T}_{\mathrm{RM}}$ cells induced infiltration of CD8 $\mathrm{T}$ cells into the vagina (Figure $\mathbf{6 b}$ ). In vaccinated mice instilled with the AI9 peptide, CD8 T cells increased significantly by 3.7 -fold compared with vaccinated 


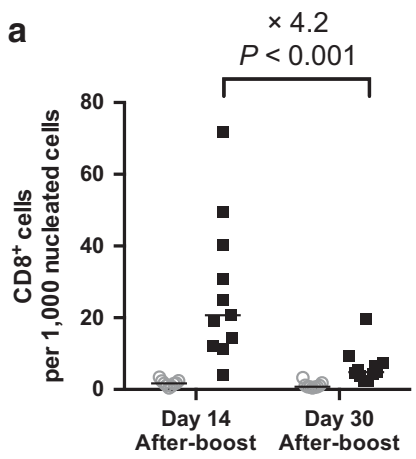

d

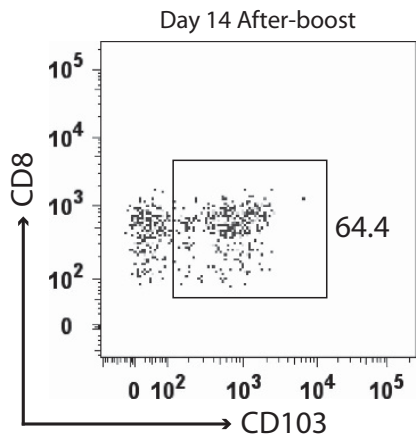

f

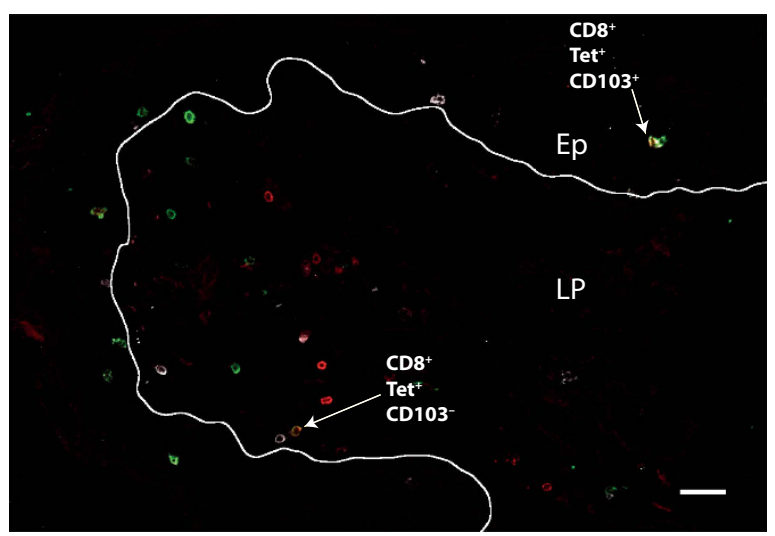

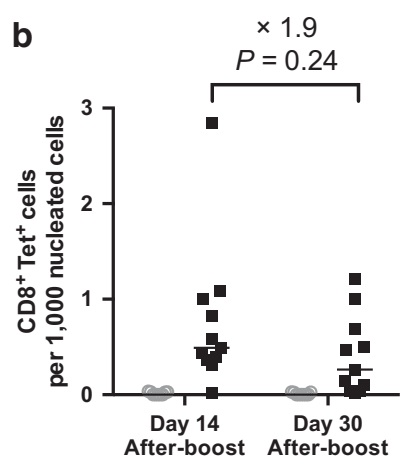
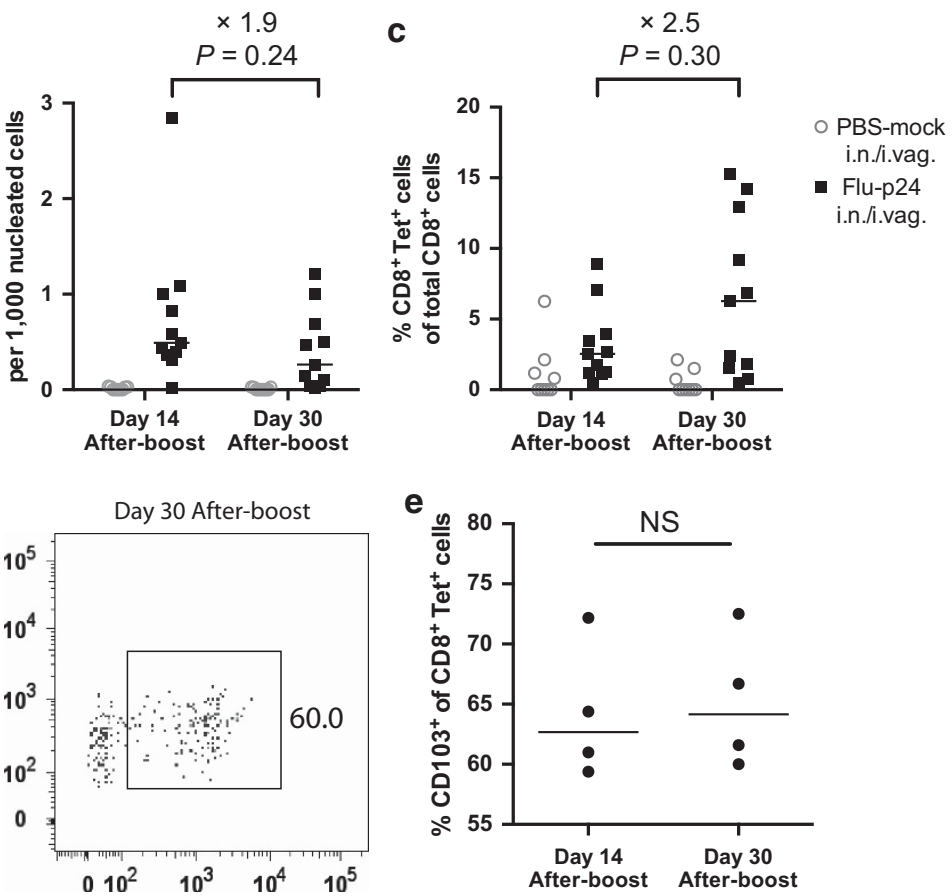

g
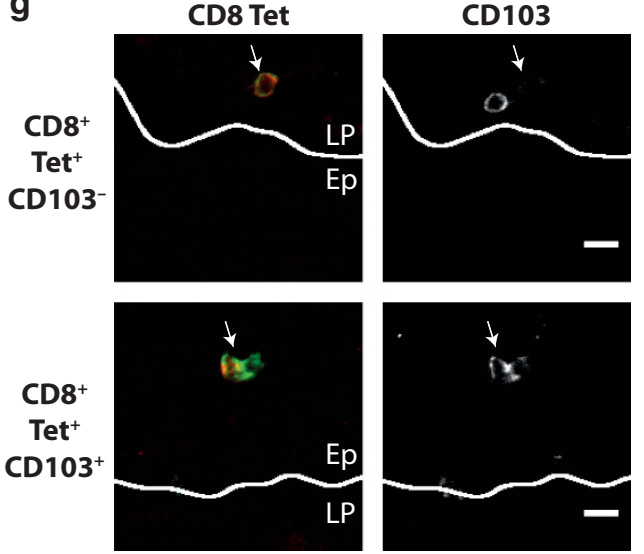

Figure 5 Maintenance of vaginal HIV-specific CD8 T cells 14 days (effector) and 30 days (memory) after the last vaccination. Quantification of (a) total CD8 T cells, (b) HIV-specific CD8 T cells, and (c) HIV-specific CD8 T cells as a percentage of total CD8 T cells in the vaginal mucosa; graphs represent data combined from two experiments $(n=9-11$ mice per treatment group). (d) CD103 expression on vaginal HIV-specific CD8 T cells; plots are representative of four independent experiments with cells pooled from three mice per treatment group. (e) Frequency of CD103 expression on vaginal HIV-specific CD8 T cells; data points represent responses pooled from three mice per treatment group. (f) Representative vaginal tissue from a Flu-p24immunized mouse displaying CD103 ${ }^{+}$or CD103 ${ }^{-}$HIV-specific CD8 T cells (arrows). Bar $=30 \mu \mathrm{m}$. (g) Magnification of CD103 ${ }^{+}$or CD103 ${ }^{-} \mathrm{HIV}^{-s p e c i f i c}$ CD8 T cells. Bar $=10 \mu \mathrm{m}$.. Bars represent median values, and $P$ values were derived from Mann-Whitney statistical testing. Ep, epithelium, i.vag., intravaginal; i.n., intranasal; LP, lamina propria; NS, not significant.

mice instilled with the LYL peptide $(P<0.0001$, Figure $6 \mathbf{c})$. The average for total CD8 $\mathrm{T}$ cells was lowest in mock-immunized mice. The increase in HIV-specific CD8 T cells in vaccinated mice receiving the AI9 peptide was not statistically significant, although median levels were elevated by 2.8 -fold compared with mice receiving the LYL peptide $(P=0.08$, Figure 6d). Vaccinated mice receiving the AI9 peptide displayed a significant correlation between HIVspecific CD8 $\mathrm{T}$ cells and total CD8 $\mathrm{T}$ cells $(P<0.05$, Figure 6e), suggesting that the magnitude of total CD8 $\mathrm{T}$-cell recruitment was regulated by the population size of $\mathrm{HIV}$-specific CD8 T cells upon reactivation. HIV-specific CD8
$\mathrm{T}$ cells showed significant upregulation of $\mathrm{CD} 44^{\text {hi }} \mathrm{CD} 62^{\text {lo }}$ phenotype upon AI9 instillation, which indicates recent T-cell activation ${ }^{44-46}$ (Figure 6f,g). Ki67 staining confirmed that the increase in total CD8 T-cell numbers was largely due to

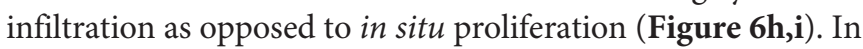
support of this, total CD8 T cells also showed an increase in CD62L (L-selectin) expression (Figure 6j,k), which is indicative of activated CD8 T-cell recruitment from peripheral circulation into sites of inflammation. ${ }^{47}$ Thus, we demonstrate here that recognition of cognate antigen by HIVspecific CD8 $\mathrm{T}_{\mathrm{RM}}$ cells resulted in the rapid recruitment of peripheral CD8 $\mathrm{T}$ cells. 
a

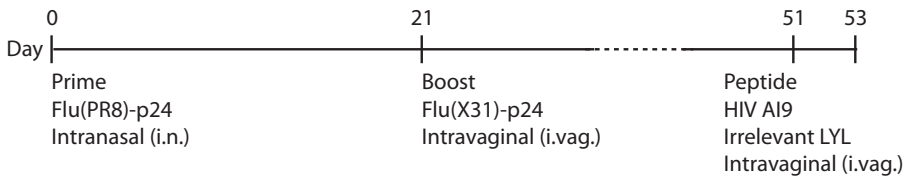

b
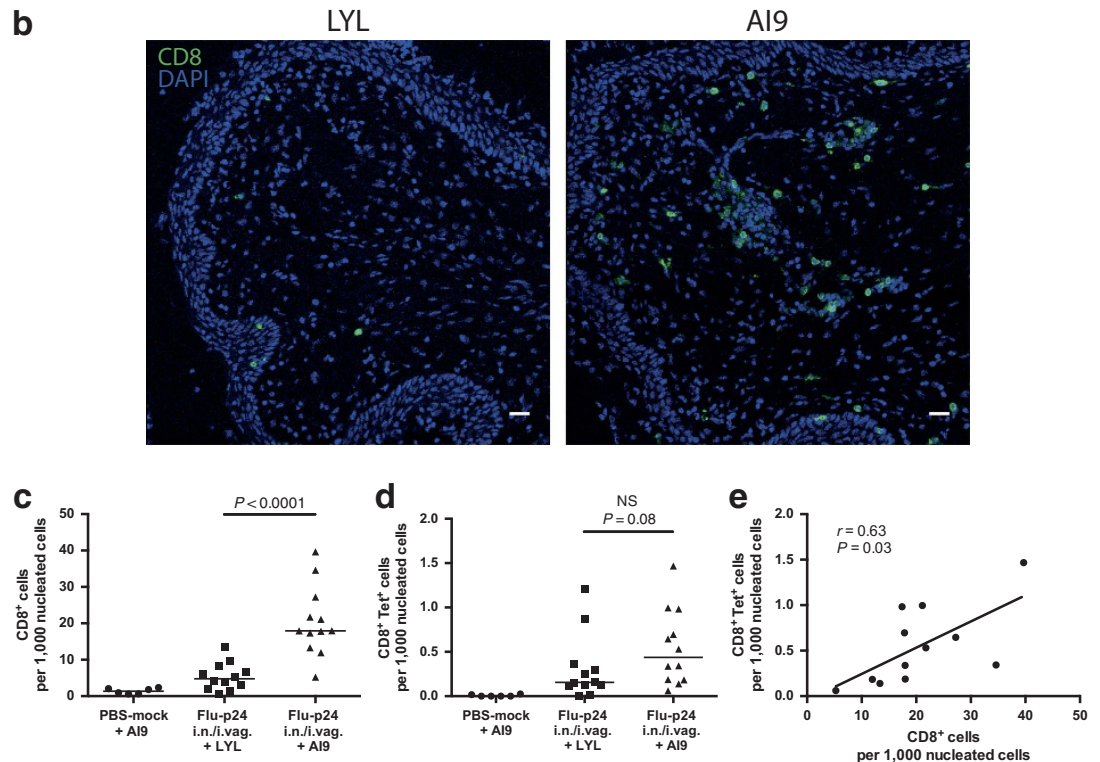

per 1,000 nucleated cells
pells

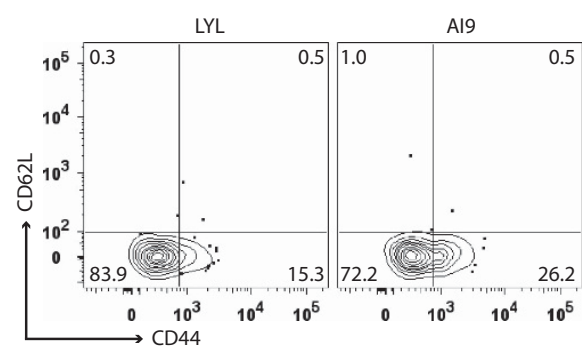

9

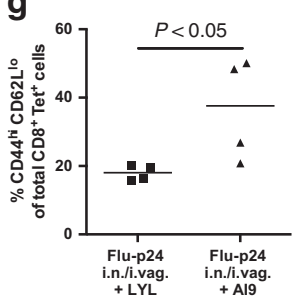

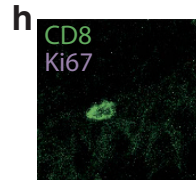
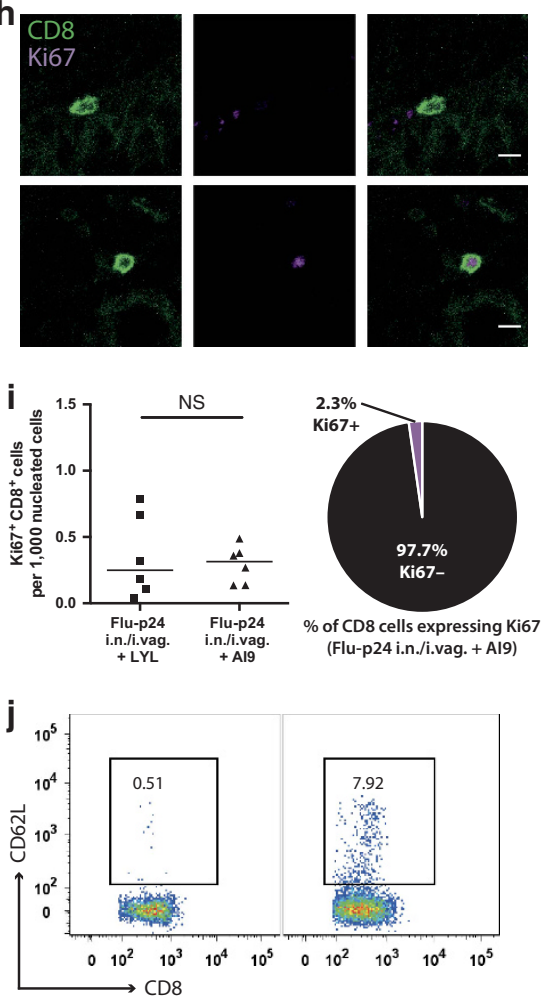

K

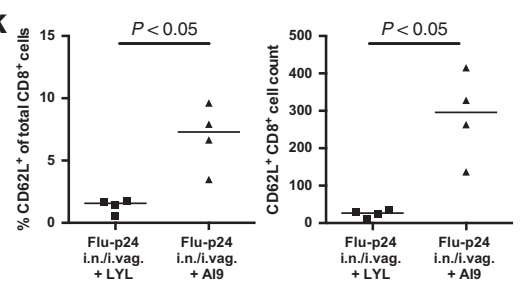

Figure 6 Tissue-wide infiltration of cytotoxic T lymphocytes (CTLs) induced by reactivation of HIV-specific CD8 $T_{\text {RM }}$ cells. (a) $T_{\text {RM }}$ reactivation of vaccinated mice by intravaginal peptide instillation. (b) Representative images of CD8 T-cell influx postpeptide instillation. Bars $=30 \mu \mathrm{m}$. Quantification of (c) total CD8 T cells and (d) HIV-specific CD8 T cells in the vaginal mucosa. (e) Correlation of HIV-specific CD8 T cells to total CD8 T cells in the vaginal mucosa of vaccinated mice receiving Al9 peptide; the $P$ value was determined by Spearman's correlation analysis. Graphs $C-E$ represent data combined from two experiments ( $n=6-12$ mice per treatment group). (f) Upregulation of CD44 ${ }^{\text {hi }}$ CD62 $\mathrm{L}^{\mathrm{lo}}$ activation phenotype in vaginal HIV-specific CD8 T cells; plots are representative of four independent experiments with cells pooled from three mice per treatment group. (g) Frequency of CD44 ${ }^{\text {hi }}$ CD62 $\mathrm{L}^{\mathrm{lo}}$ expression in vaginal HIV-specific CD8 T cells; data points represent responses pooled from three mice per treatment group. (h) Representative images of $\mathrm{Ki}^{-} 7^{-}$or $\mathrm{Ki} 67^{+} \mathrm{CD} 8 \mathrm{~T}$ cells in the vaginal mucosa post-Al9 activation. Bars $=10 \mu \mathrm{m}$. (i) Quantification of vaginal Ki67 ${ }^{+} \mathrm{CD} 8 \mathrm{~T}^{\mathrm{C}}$ cells from two experiments combined ( $n=6$ mice per treatment group). (j) CD62L expression in vaginal CD8 T cells; plots are representative of four independent experiments with cells pooled from three mice per treatment group. (k) Frequency (left) and absolute count (right) of vaginal CD8 T cells expressing CD62L; data points represent responses pooled from three mice per treatment group. Bars represent median values, and $P$ values were derived from Mann-Whitney statistical testing.

\section{Stimulation of vaginal-resident HIV-specific CD8 T cells with cognate peptide initiates tissue-wide recruitment of adaptive and innate immune cells}

Antigen recognition by CD8 $\mathrm{T}_{\mathrm{RM}}$ cells can initiate expression of adhesion molecules in inflamed tissues, which results in downstream recruitment of peripheral effector cells. ${ }^{8,14} \mathrm{We}$ analyzed vaginal tissues for the expression of vascular cell adhesion molecule-1 after reactivation of HIV-specific CD8 $\mathrm{T}_{\mathrm{RM}}$ cells. ${ }^{14}$ Intravaginal administration of the AI9 peptide increased VCAM-1 expression on vaginal endothelial vessels (Figure 7a). Conversely, mice administered with the non-relevant LYL peptide displayed low levels of VCAM-1 expression. The mean fluorescence intensity of VCAM-1 in vaccinated mice receiving the AI9 peptide was significantly increased by 1.9 -fold in contrast to vaccinated mice receiving the non-relevant LYL peptide ( $P=0.0001$, Figure $7 \mathbf{b}$ ). Mock-immunized mice receiving the AI9 peptide showed basal levels of VCAM-1 expression, indicating that reactivation of $\mathrm{HIV}$-specific CD8 $\mathrm{T}_{\mathrm{RM}}$ cells was a prerequisite for increased VCAM-1 expression.

As activation of CD8 $\mathrm{T}_{\mathrm{RM}}$ cells may lead to recruitment of multiple effector cell types into tissues, we compared the number of B cells, natural killer (NK) cells, and CD4 T cells in 
a

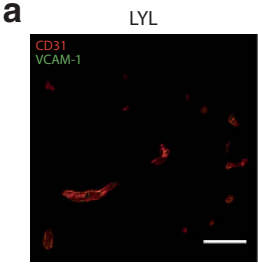

e
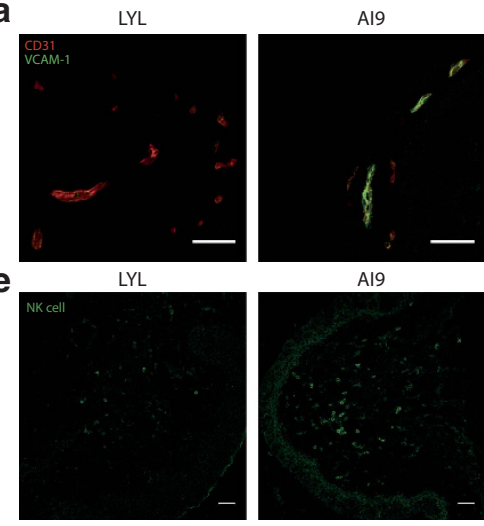

i

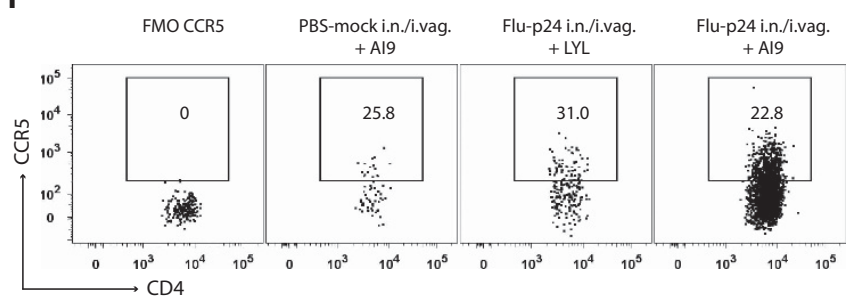

b
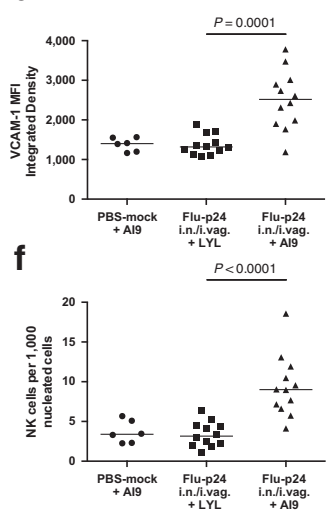
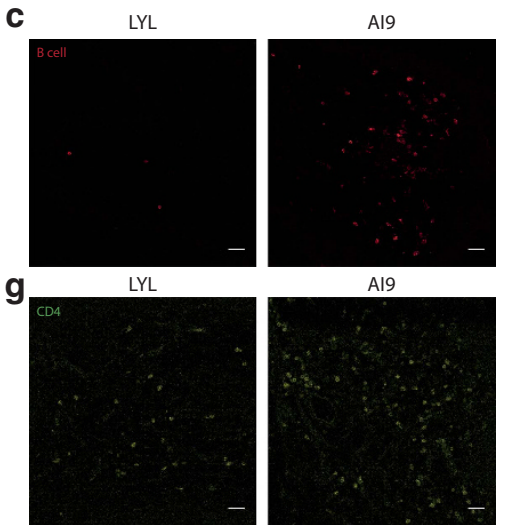

.
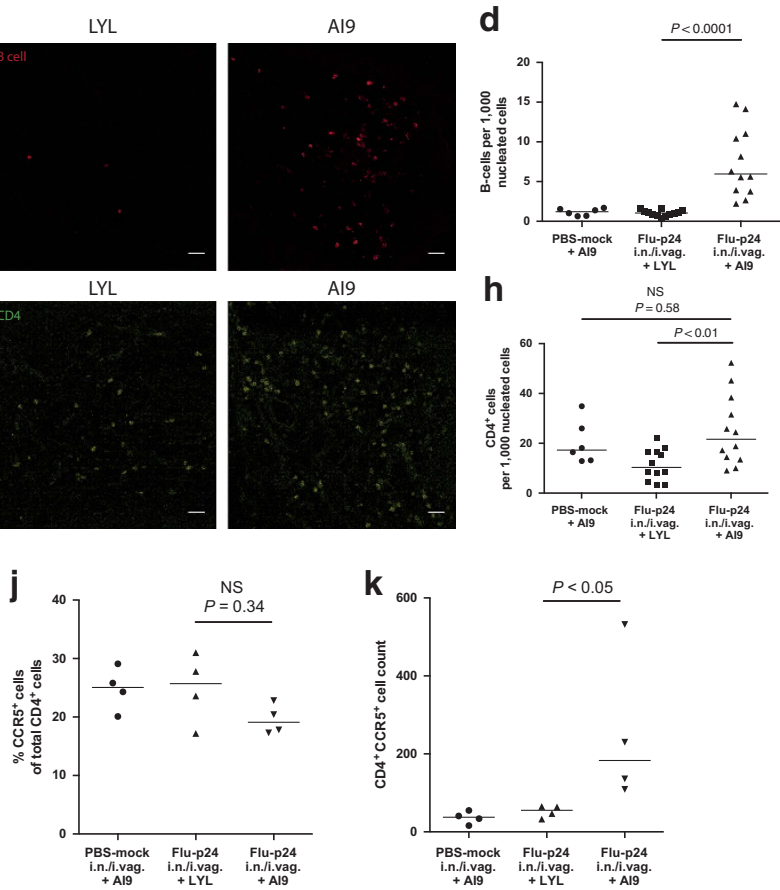

Figure 7 Induction of tissue-wide cell recruitment following reactivation of HIV-specific CD8 $\mathrm{T}_{\mathrm{RM}}$ cells. (a) Representative images of vascular cell adhesion molecule-1 (VCAM-1) expression on CD31-expressing endothelial vessels in the vaginal mucosa. Bars $=50 \mu \mathrm{M}$. (b) VCAM-1 mean fluorescence intensities. Quantification of (c and $\mathbf{d})$ B cells, (e and $\mathbf{f})$ natural killer (NK) cells, and ( $\mathbf{g}$ and $\mathbf{h})$ CD4 T cells postpeptide instillation. Bars $=30 \mu \mathrm{m}$. Graphs A-H represent data combined from two experiments ( $n=6-12$ mice per treatment group). (i) CCR5 expression in vaginal CD4 T cells postpeptide treatment; plots are representative of four independent experiments with cells pooled from three mice per treatment group. (j) Frequency and (k) absolute count of vaginal CD4 T cells expressing CCR5; data points represent responses pooled from three mice per treatment group. Bars represent median values, and $P$ values were derived from Mann-Whitney statistical testing.

the vaginal mucosa of mice following peptide stimulation of HIV-specific CD8 $\mathrm{T}_{\mathrm{RM}}$ cells. Vaginal $\mathrm{B}$ cells displayed a significant sixfold increase after activation of HIV-specific CD8 $\mathrm{T}_{\mathrm{RM}}$ cells $(P<0.0001$, Figure $7 \mathbf{c}, \mathbf{d})$, and showed significant correlation to the number of HIV-specific CD8 $\mathrm{T}_{\mathrm{RM}}$ cells $(P<0.05$, Supplementary Figure S3). Conversely, mockimmunized mice and LYL-administered mice displayed low levels of B-cell infiltration. NK cell recruitment was analyzed by NKp46 staining, which was previously demonstrated to be reflective of NK cell populations in nonlymphoid tissues. ${ }^{48,49}$ Innate $\mathrm{NK}$ cells also increased significantly by 2.8 -fold following reactivation of HIV-specific CD8 $\mathrm{T}_{\mathrm{RM}}$ cells in vaccinated mice with the AI9 peptide $(P<0.0001$, Figure 7e,f). CD4 T cells were significantly elevated by 2.1fold in vaccinated mice that received the AI9 peptide intravaginally compared with LYL-administered mice $(P<0.01$, Figure $7 \mathbf{g}, \mathbf{h})$. As an influx in CD4 $\mathrm{T}$ cells may increase the risk of HIV-1 acquisition, we examined the potential recruitment of $\mathrm{CD} 4 \mathrm{~T}$ cells that express the coreceptor $\mathrm{C}-\mathrm{C}$ motif chemokine receptor 5 (CCR5), which is required for viral fusion by most transmitted HIV-1 strains (Figure 7ik). ${ }^{50,51}$ Although reactivation of HIV-specific CD8 $\mathrm{T}_{\mathrm{RM}}$ cells induced an increase in total CD4 T cells, the percentage of CD4 $\mathrm{T}$ cells expressing CCR5 did not significantly increase (Figure 7i,j). However, the absolute cell count of CD4 T cells expressing CCR5 was significantly elevated as a result of AI9 peptide activation in vaccinated mice (Figure $7 \mathbf{k}$ ).
We also examined if whole inactivated virus (aldrithiol-2treated HIV-1) could similarly reactivate HIV-specific CD8 $\mathrm{T}_{\mathrm{RM}}$ cells in the vaginal mucosa, which would require uptake of viral particles by antigen-presenting cells and subsequent crosspresentation to CD8 $\mathrm{T}_{\mathrm{RM}}$ cells (Supplementary Figure S4A) ${ }^{52}$ With the exception of a significant increase in total CD8 T cells $(P<0.05)$ (Supplementary Figure S4D), VCAM-1, total T-cells (i.e. CD3) and HIV-specific CD8 T-cells in vaccinated mice receiving whole inactivated virus were comparable to vaccinated mice instilled with a negative control microvesicle solution (Supplementary Figures S4B,C,E). In contrast to direct peptide instillation, the stimulation of HIV-specific CD8 $\mathrm{T}_{\mathrm{RM}}$ cells by whole inactivated viral particles did not induce the associated tissue-wide responses previously observed.

These studies highlight that reactivation of HIV-specific CD8 $T_{R M}$ cells in the vaginal mucosa can be achieved by direct recognition of cognate peptide. Furthermore, this effect was highly specific to the HIV antigen and was not observed with an irrelevant antigen. Cognate antigen recognition rapidly resulted in tissue-wide recruitment of both adaptive and innate immune cells that also coincided with increased expression of addressin molecules on endothelial vessels in the vaginal mucosa.

\section{DISCUSSION}

CD8 $T_{R M}$ cells are primed to rapidly respond to localized tissue infections and the significance of $\mathrm{T}_{\mathrm{RM}}$ cells in protection against 
viral infections has been illustrated in several important studies. ${ }^{6,7,9,17-20}$ Using a prime-boost mucosal regimen involving heterologous recombinant influenza vectors expressing the HIV-1 p24 capsid protein, we demonstrate that a mucosal vaccination regimen combining intranasal and intravaginal routes induces vaginal-resident HIV-specific CD8 T cells. Furthermore, this mucosal vaccination strategy primed CD8 $\mathrm{T}$ cells to preferentially localize to the epithelial compartment of the vaginal mucosa. Established HIV-specific CD8 $\mathrm{T}_{\mathrm{RM}}$ cells could detect cognate HIV antigen intravaginally and trigger a robust anamnestic response within the vaginal mucosa. HIV-specific CD8 $\mathrm{T}_{\mathrm{RM}}$ cell reactivation resulted in increased addressin expression on vaginal endothelial vasculature. Moreover, vascular activation was coupled with heightened infiltration of both adaptive and innate immune cells.

In this study, vaginal CD8 $\mathrm{T}$ cells showed considerable difference in their spatial distribution pre- and postimmunization. Before immunization, the majority of CD8 T cells resided in the lamina propria, indicating that these populations were likely to be migratory. ${ }^{12}$ Immunization led to a significant shift in the anatomical localization of CD8 T cells, with these cells skewed toward the epithelial compartment. This redistribution may have a crucial role in intercepting early HIV transmission and for the clearance of infected cells residing in the intraepithelial compartment. Direct infection of vaginal intraepithelial CD4 $\mathrm{T}$ cells expressing CCR5 occur in the earliest phases of productive HIV infection. ${ }^{3}$ Resident dendritic cells expressing C-type lectin receptors can also bind HIV-1 by gp120 association. ${ }^{53,54}$ Similarly, epithelial Langerhan cells productively uptake HIV-1 and form infectious synapses with T cells. ${ }^{3}$ Infected cells can migrate out of the vaginal epithelial compartment, leading to subsequent viral amplification in the lamina propria or draining lymph nodes before systemic dissemination. Previous large-scale T-cell vaccine trials (STEP and Phambili) have largely focused on inducing systemic immunity towards HIV, which failed to demonstrate protection against sexually transmitted HIV. ${ }^{21-23}$ Although systemic responses induced by these vaccine regimens displayed observable magnitude and cytokine functionality, seeding of CD8 T cells into genital mucosal tissues was likely inadequate for the effective control of infection at its entry point. HIV vaccines that generate $C D 8 T_{R M}$ cells within the epithelial compartment of the female reproductive tract could circumvent the temporal delay associated with anamnestic recall of peripheral memory CD8 $\mathrm{T}$ cells, and therefore provide the rapid cell-mediated functions required to halt the earliest phases of genital HIV infection.

We show that HIV-specific CD8 T cells displayed a tissueresident phenotype in the vaginal epithelium compartment 30 days after the last immunization. This was demonstrated by the expression of CD103, a marker of CD8 T-cell maintenance in the female reproductive tract of mice and humans. ${ }^{43,55}$ Despite high and stable expression of CD103 between day 14 and 30 postimmunization shown in our studies, a modest reduction in HIV-specific CD8 T cells was observed. The magnitude in decline from 14 to 30 days after immunization of HIV-specific CD8 T cells was, however, less compared with the reduction in total vaginal CD8 $\mathrm{T}$ cells. This observation is in agreement with a recent study showing that although trafficking of total CD8 T cells into non-lymphoid tissues was elevated and indiscriminate early in vaccinia infection, only antigen-specific CD8 T cells were preferentially retained after the resolution of infection. ${ }^{56}$ Retention of $\mathrm{HIV}$-specific CD8 $\mathrm{T}$ cells in the vaginal mucosa may mirror the kinetics of CD8 $\mathrm{T}_{\mathrm{RM}}$ cells in lungs, in which a steady rate of attrition was observed over time compared with stable populations in other non-lymphoid sites such as skin. ${ }^{17,19,57}$ Despite a 1.9 -fold decrease in vaginalresident HIV-specific CD8 T cells, the remaining cells displayed the capacity for in situ reactivation following HIV peptide delivery to the vagina. Although the presence of $\mathrm{T}_{\mathrm{RM}}$ cells at 30 days after infection has been shown to predict long-term residency and protection, ${ }^{6,12,17,18,36}$ it will be important in future studies to address the degree to which even longer-term persistence of HIV-specific CD8 T cells can be generated by our vaccination model.

We expected that the reactivation of vaginal CD8 $T_{R M}$ cells would have induced rapid proliferation and peripheral recruitment of other HIV-specific CD8 T cells. An overall 2.8-fold increase in absolute numbers of HIV-specific CD8 T cells was observed; however, this was variable across the mice studied and did not reach significance. Other studies have shown that the reactivation of CD8 $\mathrm{T}_{\mathrm{RM}}$ cells induces significant accumulation of antigen-specific CD8 $\mathrm{T}$ cells in a similar 2-day period. ${ }^{8,14}$ The number of HIV-specific CD8 $\mathrm{T}_{\mathrm{RM}}$ cells before reactivation measured in our study was comparatively lower than the CD8 $\mathrm{T}_{\mathrm{RM}}$ cell densities observed in these previous studies. This difference in CD8 $\mathrm{T}_{\mathrm{RM}}$ cell quantities established within the tissue before antigen reactivation may likely influence the downstream influx rate of other immune cells, with higher densities resulting in more rapid recruitment. Future studies could more finely optimize the density of HIVspecific CD8 $\mathrm{T}_{\mathrm{RM}}$ cells within the vaginal mucosa and analyze the corresponding time course of recruitment of both HIVspecific $\mathrm{CD} 8 \mathrm{~T}_{\mathrm{RM}}$ cells and other immune cell populations after antigen exposure in the vagina.

Upon CD8 $\mathrm{T}_{\mathrm{RM}}$ cell reactivation, increased infiltration of $\mathrm{CD} 4 \mathrm{~T}$ cells was observed. We performed the peptide inoculation as a surrogate of viral reinfection, and under circumstances of an actual infection, the elevation in target CD4 T cells could inadvertently result in a more rapid dissemination of HIV within the vaginal mucosa. Our studies specifically demonstrate that absolute numbers of CCR5-expressing CD4 T cells were elevated in the vaginal mucosa postpeptide instillation, suggesting a potential increased risk of infection early after $\mathrm{CD} 8 \mathrm{~T}_{\mathrm{RM}}$ reactivation. Conversely, while an influx of activated CD4 T cells present more potential target cells, CD4 T cells may also serve to enhance viral clearance and promote cytotoxic $\mathrm{T}$ lymphocyte responses. ${ }^{9,58,59}$ In individuals acutely infected with HIV, HIV-specific CD4 T cells displaying cytolytic properties contribute to the control of viral replication. ${ }^{60}$ Although robust anti-viral responses can be achieved with CD8 
$T_{R M}$ cell reactivation, our data suggest that caution should be taken with the use of $\mathrm{T}_{\mathrm{RM}}$-based vaccine strategies against HIV. These risks and benefits of an expanded CD4 T-cell population driven by $\mathrm{CD} 8 \mathrm{~T}_{\mathrm{RM}}$ cell reactivation or the ability to selectively induce reactivation without the recruitment of HIV target cells will require further investigation.

B-cell infiltration was similarly enhanced in the vaginal mucosa, which suggests that humoral-mediated responses could also be promoted by the activation of $\mathrm{CD} 8 \mathrm{~T}_{\mathrm{RM}}$ cells. We also found that B-cell infiltration was significantly correlated to the number of HIV-specific CD8 $\mathrm{T}_{\mathrm{RM}}$ cells post-AI9 reactivation. Several studies have demonstrated strong association of anti-HIV activity in females with high levels of cervicovaginal immunoglobulin $\mathrm{G}$ or $\mathrm{A}^{61-63}$ If combined with a humoral vaccination regimen, $\mathrm{CD} 8 \mathrm{~T}_{\mathrm{RM}}$ cells could conceivably recruit $\mathrm{B}$ cells expressing HIV-specific antibodies rapidly to the vaginal mucosa after viral exposure. The observed elevation of innate NK cell numbers following CD8 $\mathrm{T}_{\mathrm{RM}}$ cell reactivation could also potentiate anti-viral defenses, whereby the distinct cytolytic capabilities of NK cells and CD8 T cells are harnessed simultaneously for the clearance of mucosal HIV infection. NK cell-mediated immunity by antibody-dependent cellular cytotoxicity likely conferred modest levels of protection in the RV144 HIV vaccine efficacy trial. ${ }^{64-66}$ Vaccine regimens that induce non-neutralizing antibody-dependent cellular cytotoxicity antibodies and concurrent seeding of mucosal CD8 $\mathrm{T}_{\mathrm{RM}}$ cells could be utilized as a strategy for rapid viral clearance in the vaginal mucosa. Furthermore, CD8 $\mathrm{T}_{\mathrm{RM}}$ cell reactivation was previously shown to significantly upregulate granzyme $\mathrm{B}$ production in NK cells. ${ }^{14}$ These observations allude to the capacity of $\mathrm{T}_{\mathrm{RM}}$-based vaccines to bridge multiple effector components and impart a synergistic effect for the potential control of mucosal HIV infection.

We hypothesized that intravaginal exposure of mice to whole inactivated HIV-1 would initiate in vivo cross-presentation of p24 Gag epitopes, mirroring the reactivation of CD8 $T_{R M}$ cells with exogenous peptides. Non-infectious HIV-1 particles can be acquired and cross-presented by antigen-presenting cells, resulting in the activation of antigen-specific $\mathrm{T}$ cells from HIV-infected individuals. ${ }^{52}$ However, the tissue-wide activation state was not induced with whole inactivated virus, and we attribute this divergence to several key differences that exist with the peptide-based reactivation model. In contrast to the intravaginal peptide instillation, the maximum practical dose of whole inactivated virus preparations used in our studies contained 17-fold less cognate p24 Gag antigen. This alludes to a likely physiological threshold of cognate antigen concentration required to effectively stimulate $\mathrm{CD} 8 \mathrm{~T}_{\mathrm{RM}}$ cells. We also speculate that the activation kinetics induced by cross-presentation of whole inactivated virus antigen in antigen-presenting cells would be comparatively delayed due to additional intracellular antigen processing pathways, as opposed to the direct major histocompatibility complex-I binding of exogenous peptides. ${ }^{67}$ Lastly, the use nonreplicating viral particles with static antigenic loads would likely be less efficient in stimulating CD8 $\mathrm{T}_{\mathrm{RM}}$ cells compared with live replication-competent viruses, which would exponentially increase the amount of antigen available. Future nonhuman primate studies utilizing live viral challenge models are more likely to mimic transmission of HIV-1 in humans and the corresponding level of protection conferred by $\mathrm{CD} 8 \mathrm{~T}_{\mathrm{RM}}$ cells.

In summary, we demonstrate that vaccination strategies using live viral vectors result in the establishment of HIVspecific CD8 $T_{R M}$ cells at the frontline barrier of the vaginal mucosa. The anatomical localization of these cells combined with their ability to rapidly induce recruitment in both innate and adaptive immune arms may provide heightened local surveillance and immunity for the protection against vaginal HIV-1 exposure.

\section{METHODS}

Mice immunizations. All animal procedures were approved by the University of Melbourne Animal Ethics Committee. Female BALB/c mice at 6-8 weeks of age were used in all experiments. H1N1 (Flu(PR8)-p24) or H3N2 (Flu(X31)-p24) subtype recombinant influenza viruses expressing the HIV-1 subtype B Gag p24 capsid gene were developed as described previously. ${ }^{32}$ Mice were anesthetized by isoflurane inhalation before immunizations. Intranasal immunization was performed with $10^{5}$ p.f.u. $/ 50 \mu$ l of Flu(PR8)-p24 virus. Five days before intravaginal immunization, mice were injected subcutaneously with $2 \mathrm{mg}$ of medroxyprogesterone acetate (Pharmacia and Upjohn, Pfizer Inc., NY) in $100 \mu$ l phosphate-buffered saline (PBS). Mice were intravaginally immunized by atraumatic instillation of Flu(X31)-p24 virus at $10^{7}$ p.f.u. $/ 30 \mu$ l. For the vector-only control group, matching doses of wild-type PR8 and X31 influenza viruses were used, which were generated as described previously. ${ }^{32}$

Lymphocyte isolation and tetramer staining. BALs ( $3 \mathrm{ml}$ per mouse), whole blood ( $100 \mu \mathrm{l}$ per mouse), and vaginal tissues were pooled from three mice per group. Lymphocyte isolation was performed as described previously. ${ }^{32}$ Vaginal $\mathrm{T}$ cells were enriched using a negative selection EasySep Mouse T Cell Isolation Kit (STEMCELL Technologies, Vancouver, BC, Canada), as per the manufacturer's protocol. Cells were stained with a viability dye (Life Technologies, Thermo Fisher Scientific, Waltham, MA) for $30 \mathrm{~min}$ at room temperature and washed once, and Fc blocked with a CD16/32 antibody (93; BioLegend, San Diego, CA) for $10 \mathrm{~min}$ at room temperature. Cells were subsequently incubated for $1 \mathrm{~h}$ at room temperature with $1.25 \mu \mathrm{g} / \mathrm{ml}$ of phycoerythrin-conjugated major histocompatibility complex-I H2- $\mathrm{K}^{\mathrm{D}}$ AI9 tetramer or an irrelevant phycoerythrin-conjugated major histocompatibility complex-I H2-K ${ }^{\mathrm{D}}$ TUM tetramer (University of Melbourne, Melbourne, VIC, Australia). After tetramer staining, surface staining of cells was performed for $30 \mathrm{~min}$ at room temperature using the following surface antibodies: CD3 (17A2; BioLegend), CD4 (GK1.5; BD, Becton, Dickinson and Company, Franklin Lakes, NJ), CD8 (53-6.7; BD), CD103 (2E7; Miltenyi Biotec, Bergisch Gladbach, Germany), CD45 (30-F11; BD), CD44 (IM7; BD), and CD62L (MEL14; BioLegend). CD4 T-cell CCR5 coreceptor was stained using the CD195 (7A4; eBioscience, Thermo Fisher Scientific) antibody for $30 \mathrm{~min}$ at $37^{\circ} \mathrm{C}$. Cells were washed two times with PBS and fixed with Stabilizing Fixative solution (BD). Flow cytometry data were acquired with a BD LSRFortessa (Becton, Dickinson and Company) and analyzed with FlowJo 9.8.3 (Ashland, OR).

Whole tissue IST staining. IST staining was performed as previously described previously. ${ }^{38}$ Briefly, vaginal tissues were collected in RF5 media (RPMI1640, 5\% fetal calf serum, $10 \mathrm{~mm}$ HEPES; Life Technologies). Sectioned tissues of $2 \mathrm{~mm}$ in length were placed in $200 \mu \mathrm{l}$ solution of PBS with $2 \%(\mathrm{v} / \mathrm{v})$ fetal calf serum (Life Technologies) and $2 \%$ (v/v) normal goat serum (Jackson ImmunoResearch, West Grove, PA). Tissues were aspirated and replaced with $1.25 \mu \mathrm{g} / \mathrm{ml}$ of tetramer solution for $16 \mathrm{~h}$ at $4{ }^{\circ} \mathrm{C}$. Tissues were then washed with cold PBS for 
$20 \mathrm{~min}$ three times. Tissues were fixed in $2 \%(\mathrm{v} / \mathrm{v})$ paraformaldehyde solution (Electron Microscopy Sciences, Hatfield, PA) for $2.5 \mathrm{~h}$ at $4{ }^{\circ} \mathrm{C}$. Fixed tissues were washed once with cold PBS solution for $20 \mathrm{~min}$, and placed in $30 \%(\mathrm{w} / \mathrm{v})$ sucrose solution (Sigma, St. Louis, MO) at $4{ }^{\circ} \mathrm{C}$ until sunken. Tissues were snap frozen in OCT compound (Sakura Finetek USA, Torrance, CA) and stored at $-80^{\circ} \mathrm{C}$.

Immunofluorescence tissue section staining. Seven micrometers of sectioned tissues were fixed in cold acetone solution (Sigma) for $10 \mathrm{~min}$. Tissues were rehydrated with PBS for $10 \mathrm{~min}$ and subsequently blocked with 5\% (w/v) bovine serum albumin (Sigma) for $1 \mathrm{~h}$. Antibodies were diluted in PBS supplemented with 2\% (v/v) normal goat serum (Jackson ImmunoResearch). To amplify the tetramer-PE signal, tissues were stained sequentially with rabbit polyclonal anti-PE antibodies (Novus Biologicals, Littleton, CO) and a secondary goat anti-rabbit immunoglobulin G Alexa Fluor 555 antibody (Life Technologies). Cell staining was performed using the following surface antibodies: CD8 (53.6.7; BioLegend), CD4 (GK1.5 or RM4-5; BioLegend), CD335/NKp46 (29A1.4; BioLegend), CD103 (2E7; BioLegend), CD45R/B220 (RA3-6B2; BioLegend), and Ki67 (11F6; BioLegend). Addressin expression on endothelial vessels was detected using VCAM-1/CD106 (429; BioLegend) and CD31 (MEC13.3; BioLegend) antibodies. Secondary staining of nonconjugated primary antibodies was performed with goat anti-rat immunoglobulin G Alexa Fluor 555/647 (Life Technologies) or goat anti-hamster Alexa Fluor 647 antibodies (BioLegend). Nuclear staining was performed with DAPI (4',6-diamidino-2-phenylindole) stain (Life Technologies). Slides were sealed with ProLong Diamond Antifade Mountant (Life Technologies).

Confocal image analysis. Tiled images were captured on a Zeiss LSM710 instrument (Oberkochen, Germany). Postprocessing of confocal images was performed with ImageJ v.2.0.0 (National Institutes of Health, Bethesda, MD). Images were split into separate fluorescence channels and transformed into binary 8-bit images using the "Threshold" and "Watershed" function. Colocalization of signals was performed using the "Image Calculator" function. A triple colocalization signal criterion was used in identifying tetramerpositive $\mathrm{CD} 8 \mathrm{~T}$ cells $(\mathrm{CD} 8 / \mathrm{Tetramer} / \mathrm{DAPI}), \mathrm{Ki}^{+}{ }^{+}$cells $(\mathrm{CD} 8 / \mathrm{Ki67} /$ DAPI), or $\mathrm{CD} 103^{+}$cells (CD103/CD8/Tetramer). Enumeration of NK cells, B cells, CD8 T cells, or CD4 T cells was performed by a double colocalization signal criterion (surface signal/DAPI). Cells were quantified as a proportion of 1,000 nucleated cells. Cell quantification in each mouse was performed on an average of 16 long-stitched images $(3 \times 1$ field of view, $\times 20$ magnification, 12 Z-stacks). Spatial localization of CD8 $\mathrm{T}$ cells was performed by the manual creation of an image mask on the DAPI fluorescence image, demarcating the lamina propria and epithelial compartments of the vaginal mucosa. To assess VCAM-1 expression on endothelial cells, images were split into the VCAM-1 or CD31 fluorescence channels. The CD31 fluorescence channel was transformed into a binary 8-bit image using the "Threshold" function for the identification of endothelial vessels. The CD31 image was utilized as a mask and overlapping VCAM-1 expression was measured by use of the "Set Measurements: Redirect To" function. Mean fluorescence intensities of VCAM-1 were measured as integrated densities (mean of VCAM-1 fluorescence $\times$ area of VCAM-1/CD31 endothelial vessel).

Reactivation of HIV-specific CD8 $\mathrm{T}_{\mathrm{RM}}$ by intravaginal instillation of peptide or whole inactivated virus. The $\mathrm{H} 2-\mathrm{K}^{\mathrm{D}}$-restricted HIV p24 epitope AI9 (AMQMLKETI) or the irrelevant $\mathrm{H} 2-\mathrm{K}^{\mathrm{D}}$-restricted LYL (LYLVCGERL) peptide was diluted in $30 \%$ (v/v) dimethyl sulfoxide to a final concentration of $2.5 \mu \mathrm{g} / \mu \mathrm{l}$. Aldrithiol-2-inactivated whole HIV1 subtype B preparations containing the AI9 epitope $(0.1 \mu \mathrm{g} / \mu \mathrm{l}$ of $\mathrm{p} 24$ capsid) or control microvesicles were kindly provided by Jeff Lifson (AIDS Vaccine Program, National Cancer Institute). ${ }^{52}$ Vaginal canals of anesthetized mice were gently swabbed with calcium alginate swabs (Puritan) moistened with PBS. Twenty microliters of peptide solution or $30 \mu \mathrm{l}$ of inactivated virus were administered intravaginally by atraumatic instillation.

Statistical analysis. Analyses were performed using Prism 6.0 (GraphPad Software, La Jolla, CA). Data sets were analyzed by a Kruskal-Wallis test, followed by Mann-Whitney tests. A Bonferroni adjustment was used to set a new $P$ value level $(0.05 \div n$; where $n$ is the number of comparisons made) for these analyses; $P$ values $<(0.05 \div n)$ were considered significant. Correlation $P$ values were determined using a Spearman's test.

SUPPLEMENTARY MATERIAL is linked to the online version of the paper at http://www.nature.com/mi

\section{ACKNOWLEDGMENTS}

This research was supported by the Australian Research Council and National Health and Medical Research Council, Australia.

\section{AUTHOR CONTRIBUTIONS}

H.-X.T., S.J.K., and R.D.R. designed the experiments; H.-X.T., A.K.W., S.J, R.E., and J.J.G. performed experiments; D.M. established the IST staining technique; H.-X.T., S.J.K., and R.D.R. analysed and interpreted the data; H.-X.T., S.J.K., and R.D.R. wrote the manuscript.

\section{DISCLOSURE}

The authors declare no conflict of interest.

(c) 2018 Society for Mucosal Immunology

\section{REFERENCES}

1. Zhang, Z.-Q. et al. Sexual transmission and propagation of SIV and HIV in resting and activated CD4 + T cells. Science 286, 1353-1357 (1999).

2. Miller, C.J. et al. Propagation and dissemination of infection after vaginal transmission of simian immunodeficiency virus. J. Virol. 79, 9217-9227 (2005).

3. Hladik, F. et al. Initial events in establishing vaginal entry and infection by human immunodeficiency virus type-1. Immunity 26, 257-270 (2007).

4. Mueller, S.N., Gebhardt, T., Carbone, F.R. \& Heath, W.R. Memory T cell subsets, migration patterns, and tissue residence. Annu. Rev. Immunol. 31, 137-161 (2013).

5. Masopust, D. et al. Dynamic T cell migration program provides resident memory within intestinal epithelium. J. Exp. Med. 207, 553-564 (2010).

6. Gebhardt, T. et al. Memory T cells in nonlymphoid tissue that provide enhanced local immunity during infection with herpes simplex virus. Nat. Immunol. 10, 524-530 (2009).

7. Jiang, X. et al. Skin infection generates non-migratory memory CD8 + TRM cells providing global skin immunity. Nature 483, 227-231 (2012).

8. Schenkel, J.M., Fraser, K.A., Vezys, V. \& Masopust, D. Sensing and alarm function of resident memory CD8 + T cells. Nat. Immunol. 14, 509-513 (2013).

9. lijima, N. \& Iwasaki, A. A local macrophage chemokine network sustains protective tissue-resident memory CD4 Tcells. Science 346, 93-98 (2014).

10. Masopust, D., Vezys, V., Marzo, A.L. \& Lefrançois, L. Preferential localization of effector memory cells in nonlymphoid tissue. Science 291, 2413-2417 (2001)

11. Reinhardt, R.L., Khoruts, A., Merica, R., Zell, T. \& Jenkins, M.K. Visualizing the generation of memory CD4 T cells in the whole body. Nature 410, 101-105 (2001).

12. Gebhardt, T. et al. Different patterns of peripheral migration by memory CD4 + and CD8 + T cells. Nature 477, 216-219 (2011).

13. Ariotti, S. et al. Skin-resident memory CD 8 + Tcells trigger a state of tissuewide pathogen alert. Science 346, 101-105 (2014).

14. Schenkel, J.M. et al. Resident memory CD8 T cells trigger protective innate and adaptive immune responses. Science 346, 98-101 (2014).

15. Zaid, A. et al. Persistence of skin-resident memory $\mathrm{T}$ cells within an epidermal niche. Proc. Natl Acad. Sci. USA 111, 5307-5312 (2014). 
16. Ariotti, S. et al. Tissue-resident memory CD8 + Tcells continuously patrol skin epithelia to quickly recognize local antigen. Proc. Natl Acad. Sci. USA 109, 19739-19744 (2012).

17. Mackay, L.K. et al. Long-lived epithelial immunity by tissue-resident memory T (TRM) cells in the absence of persisting local antigen presentation. Proc. Natl Acad. Sci. USA 109, 7037-7042 (2012).

18. Shin, H. \& Iwasaki, A. A vaccine strategy that protects against genital herpes by establishing local memory T cells. Nature 491, 463-467 (2012).

19. Wu, T. et al. Lung-resident memory CD8 T cells (TRM) are indispensable for optimal cross-protection against pulmonary virus infection. J. Leukoc. Biol. 95, 215-224 (2014).

20. Morabito, K.M. et al. Intranasal administration of RSV antigen-expressing MCMV elicits robust tissue-resident effector and effector memory CD8 + T cells in the lung. Mucosal Immunol. 10, 545-554 (2016).

21. Buchbinder, S.P. et al. Efficacy assessment of a cell-mediated immunity HIV-1 vaccine (the Step Study): a double-blind, randomised, placebocontrolled, test-of-concept trial. Lancet 372, 1881-1893 (2008).

22. McElrath, M.J. et al. HIV-1 vaccine-induced immunity in the test-ofconcept Step Study: a case-cohort analysis. Lancet 372, 1894-1905 (2008).

23. Gray, G.E. et al. Safety and efficacy of the HVTN 503/Phambili Study of a clade-B-based HIV-1 vaccine in South Africa: a double-blind, randomised, placebo-controlled test-of-concept phase 2b study. Lancet Infect. Dis. 11, 507-515 (2011).

24. Hammer, S.M. et al. Efficacy trial of a DNA/rAd5 HIV-1 preventive vaccine. N. Engl. J. Med. 369, 2083-2092 (2013).

25. Rolland, M. et al. Genetic impact of vaccination on breakthrough HIV-1 sequences from the STEP trial. Nat. Med. 17, 366-371 (2011).

26. Hertz, T. et al. A study of vaccine-induced immune pressure on breakthrough infections in the Phambili phase $2 \mathrm{~b} H \mathrm{HIV}-1$ vaccine efficacy trial. Vaccine 34, 5792-5801 (2016).

27. Reynolds, M.R. et al. CD8 + T-lymphocyte response to major immunodominant epitopes after vaginal exposure to simian immunodeficiency virus: too late and too little. J. Virol. 79, 9228-9235 (2005).

28. Davenport, M.P., Ribeiro, R.M. \& Perelson, A.S. Kinetics of virus-specific CD8 + T cells and the control of human immunodeficiency virus infection. J. Virol. 78, 10096-10103 (2004).

29. Hansen, S.G. et al. Profound early control of highly pathogenic SIV by an effector memory T-cell vaccine. Nature 473, 523-527 (2011).

30. Hansen, S.G. et al. Effector memory T cell responses are associated with protection of rhesus monkeys from mucosal simian immunodeficiency virus challenge. Nat. Med. 15, 293-299 (2009).

31. Kaul, R. et al. HIV-1-specific mucosal CD8 + lymphocyte responses in the cervix of HIV-1-resistant prostitutes in Nairobi. J. Immunol. (Baltimore Md: 1950) 164, 1602-1611 (2000).

32. Tan, H.-X. et al. Recombinant influenza virus expressing HIV-1 p24 capsid protein induces mucosal HIV-specific CD8 T-cell responses. Vaccine 34 , 1172-1179 (2016)

33. Garulli, B., Di Mario, G., Stillitano, M.G., Kawaoka, Y. \& Castrucci, M.R. Exploring mucosal immunization with a recombinant influenza virus carrying an HIV-polyepitope in mice with pre-existing immunity to influenza. Vaccine 32, 2501-2506 (2014).

34. Li, Z. et al. Novel vaccination protocol with two live mucosal vectors elicits strong cell-mediated immunity in the vagina and protects against vaginal virus challenge. J. Immunol. 180, 2504-2513 (2008).

35. Çuburu, N. et al. Intravaginal immunization with HPV vectors induces tissue-resident CD8 + T cell responses. J. Clin. Invest. 122, 4606-4620 (2012).

36. Çuburu, N. et al. Topical herpes simplex virus 2 (HSV-2) vaccination with human papillomavirus vectors expressing gB/gD ectodomains induces genital-tissue-resident memory CD8 + Tcells and reduces genital disease and viral shedding after HSV-2 challenge. J. Virol. 89, 83-96 (2015).

37. Steinert, E.M. et al. Quantifying memory CD8 T cells reveals regionalization of immunosurveillance. Cell 161, 737-749 (2015).

38. Schenkel, J.M. et al. IL-15-independent maintenance of tissue-resident and boosted effector memory CD8 T cells. J. Immunol. 196, 3920-3926 (2016).

39. Skinner, P.J. \& Haase, A.T. In situ staining using MHC class I tetramers. Curr. Protoc. Immunol. 64, 17.4.1-17.4.9 (2005).
40. Tjernlund, A. et al. In situ detection of Gag-specific CD8 + cells in the Gl tract of SIV infected rhesus macaques. Retrovirology 7, 12 (2010).

41. Alon, R., Bayer, E.A. \& Wilchek, M. Cell adhesion to streptavidin via RGDdependent integrins. Eur. J. Cell Biol. 60, 1-11 (1993).

42. Chandra, N. et al. Depot medroxyprogesterone acetate increases immune cell numbers and activation markers in human vaginal mucosal tissues. AIDS Res. Hum. Retroviruses 29, 592-601 (2013).

43. Schön, M.P. et al. Mucosal $T$ lymphocyte numbers are selectively reduced in integrin ae (CD103)-deficient mice. J. Immunol. 162, 66416649 (1999).

44. Kaech, S.M. \& Ahmed, R. Memory CD8 + T cell differentiation: initial antigen encounter triggers a developmental program in naïve cells. Nat. Immunol. 2, 415 (2001).

45. Zajac, A.J. et al. Viral immune evasion due to persistence of activated Tcells without effector function. J. Exp. Med. 188, 2205-2213 (1998).

46. Nguyen, H.H. et al. Naïve CD8 ${ }^{+}$T cell derived tumor-specific cytotoxic effectors as a potential remedy for overcoming TGF- $\beta$ immunosuppression in the tumor microenvironment. Sci. Rep. 6, srep28208 (2016).

47. Mohammed, R.N. et al. L-selectin is essential for delivery of activated CD8 + Tcells to virus-infected organs for protective immunity. Cell Rep. 14, 760-771 (2016).

48. Walzer, T. et al. Identification, activation, and selective in vivo ablation of mouse NK cells via NKp46. Proc. Natl Acad. Sci. USA 104, 3384-3389 (2007).

49. Luci, C. et al. NKp46 + innate lymphoid cells dampen vaginal CD8 T cell responses following local immunization with a cholera toxin-based vaccine. PLOS ONE 10, e0143224 (2015).

50. Keele, B.F. et al. Identification and characterization of transmitted and early founder virus envelopes in primary HIV-1 infection. Proc. Natl Acad. Sci. USA 105, 7552-7557 (2008).

51. Salazar-Gonzalez, J.F. et al. Genetic identity, biological phenotype, and evolutionary pathways of transmitted/founder viruses in acute and early HIV-1 infection. J. Exp. Med. 206, 1273-1289 (2009).

52. Larsson, M. et al. Activation of HIV-1 specific CD4 and CD8 T cells by human dendritic cells: roles for cross-presentation and non-infectious HIV1 virus. AIDS Lond. Engl. 16, 1319-1329 (2002).

53. Geijtenbeek, T.B.H. et al. DC-SIGN, a dendritic cell-specific HIV-1-binding protein that enhances trans-infection of T cells. Cell 100, 587-597 (2000).

54. Cameron, P.U. et al. Dendritic cells exposed to human immunodeficiency virus type- 1 transmit a vigorous cytopathic infection to CD4 + T cells. Science 257, 383-387 (1992).

55. Kiravu, A. et al. Evaluation of CD103 ( $\alpha E \beta 7)$ integrin expression by CD8 T cells in blood as a surrogate marker to predict cervical T cell responses in the female genital tract during HIV infection. Clin. Immunol. 141, 143-151 (2011).

56. Khan, T.N., Mooster, J.L., Kilgore, A.M., Osborn, J.F. \& Nolz, J.C. Local antigen in nonlymphoid tissue promotes resident memory CD8 + T cell formation during viral infection. J. Exp. Med. 213, 951966 (2016).

57. Slütter, B. et al. Dynamics of influenza-induced lung-resident memory $T$ cells underlie waning heterosubtypic immunity. Sci. Immunol. 2, eaag2031 (2017).

58. Nakanishi, Y., Lu, B., Gerard, C. \& Iwasaki, A. CD8 + T lymphocyte mobilization to virus-infected tissue requires CD4 + T-cell help. Nature 462, 510-513 (2009).

59. Teijaro, J.R. et al. Tissue-retentive lung memory CD4 T cells mediate optimal protection to respiratory virus infection. J. Immunol. (Baltimore Md: 1950) 187, 5510-5514 (2011).

60. Soghoian, D.Z. et al. HIV-specific cytolytic CD4 T cell responses during acute HIV infection predict disease outcome. Sci. Transl. Med. 4, 123ra25123ra25 (2012).

61. Ghosh, M. et al. Anti-HIV activity in cervical-vaginal secretions from HIVpositive and -negative women correlate with innate antimicrobial levels and IgG antibodies. PLOS ONE 5, e11366 (2010).

62. Tudor, D. et al. HIV-1 gp41-specific monoclonal mucosal IgAs derived from highly exposed but lgG-seronegative individuals block HIV-1 epithelial transcytosis and neutralize CD4 + cell infection: an IgA gene and functional analysis. Mucosal Immunol. 2, 412-426 (2009). 
63. Choi, R.Y. et al. Cervicovaginal HIV-1-neutralizing immunoglobulin A detected among HIV-1-exposed seronegative female partners in HIV-1discordant couples. AIDS 26, 2155-2163 (2012).

64. Rerks-Ngarm, S. et al. Vaccination with ALVAC and AIDSVAX to prevent HIV-1 infection in Thailand. N. Engl. J. Med. 361, 2209-2220 (2009).

65. Haynes, B.F. et al. Immune-correlates analysis of an HIV-1 vaccine efficacy trial. N. Engl. J. Med. 366, 1275-1286 (2012).
66. Chung, A.W. et al. Polyfunctional Fc-effector profiles mediated by lgG subclass selection distinguish RV144 and VAX003 vaccines. Sci. Transl. Med. 6, 228ra38-228ra38 (2014).

67. De Bruijn, M.L.H. et al. Peptide loading of empty major histocompatibility complex molecules on RMA-S cells allows the induction of primary cytotoxic T lymphocyte responses. Eur. J. Immunol. 21, 2963-2970 (1991). 